\title{
Review Paper: Studying Balance in Deaf People: A Systematic Review Study
}

\author{
Ali Asghar Norasteh ${ }^{1},{ }^{*}$ Hamed Zarei ${ }^{1}$
}

1. Department of Corrective Exercises and Sports Injury, Faculty of Physical Education \& Sport Sciences, University of Guilan, Rasht, Iran.

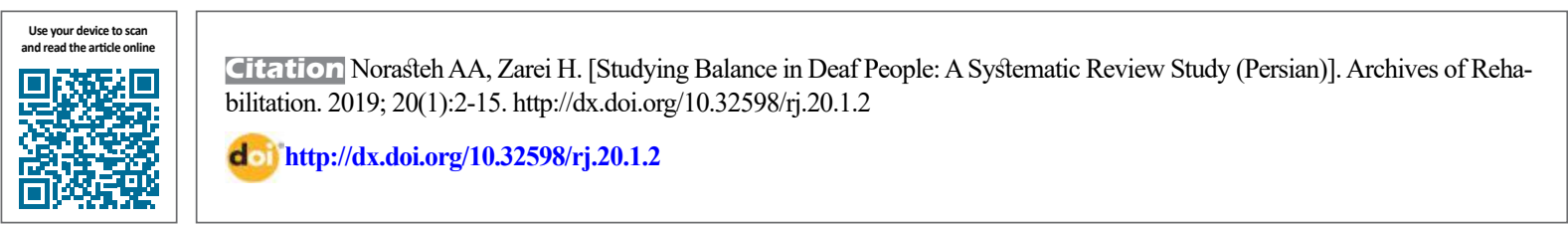

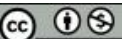

Received: 23 Nov 2018

Accepted: 09 Feb 2019

Available Online: 01 Apr 2019

\section{ABSTRACT}

Objective Damage to parts of the vestibulocochlear nerve not only results in sensory-neural hearing loss and deafness but may also lead to balance problems. This is the reason why the hearing-impaired and deaf people are struggling with balance problems. Given the ever-increasing development of the adaptive sports, specific to the deaf, who are considered as part of the active individuals in the community, detailed studies are required on the balance of the deaf, which is an important part of routine activities and sports performances. Therefore, this study generally aims to investigate the balance skills of the deaf in comparison to their normal counterparts. Materials \& Methods In this study, a comprehensive review on 'the balance of the deaf' has been carried out by searching English databases, such as Science Direct, PubMed, Cochrane review, TRIP, PEDro, EMBASE, CINAHL, and Google Scholar, for the following keywords: "Deaf", "Postural control in Deaf Hearing Loss", "Deafness", "Balance" and "Balance in Deaf". In addition, the Persian databases, such as Magiran, Irandoc, IranMedex, MedLib, Sid were searched for the following Persian equivalent keywords: Balance in the deaf, postural control in the deaf, deaf, congenital deafness and balance, from 1932 to June 2018. Moreover, manual searching and full reviewing of resources of the articles were carried out to find the respective articles. Articles were narrowed down and sorted out by the titles such as the English language, Persian language, Human, original article, and review article. After collecting the search results, the titles and abstracts of the articles were studied. If the articles met the inclusion and exclusion criteria, their results would be employed in the review study; otherwise, they would be excluded.

Results Based on the criteria and objectives of the research, 51 articles were selected. Forty-eight papers were provided in full text and the rest were summarized. In general, the balance of the deaf was investigated in three different respects of 1) a comparison of the balance of the deaf with that of the normal counterparts, 2) the effect of age on the balance of the deaf, and 3) the effect of the training protocols on the balance of the deaf. Conclusion The deaf people appear to struggle with balance problems when the vestibular system information is the only sensory source available; however, when the information of the proprioception and vision systems is available, there can be no balance problem as compared to normal counterparts. Several studies have also shown that the deaf, as they age, make up for the balance impairment resulting from vestibular impairments with proprioception and vision systems; it also appears that their proprioception and vision systems are better than or equal to those of their normal counterparts. However, it has not been determined yet that by increasing age in the deaf people, which proprioception system dominates and contributes to maintaining the balance of the deaf better than other systems. Furthermore, a review of studies has shown that all training programs and rehabilitation protocols have positive effects on the balance of the deaf. However, it has not been determined yet which training programs have a long and lasting effect on the balance of the deaf, and few studies have been carried out in this area. In addition, the same balance tests are used in all community groups, which may be inappropriate for measuring the balance in deaf people, since it is still not clear which motion strategies have been employed by the deaf to maintain balance. Therefore, to reach the final conclusion about the balance of the deaf people, further studies should be conducted on the proper tests to measure the balance of the deaf so that accurate and high-quality reports on their balance can be obtained.
Keywords:

Balance, Deaf, Postural control, Vestibulocochlear dysfunction \section{that accurate and high-quality reports on their balance can beobtained.}

\section{* Corresponding Author:}

Hamed Zarei, MSc.

Address: Department of Corrective Exercises and Sports Injury, Faculty of Physical Education \& Sport Sciences, University of Guilan, Rasht, Iran. Tel: +98 (939) 5995280

E-Mail: zareei.h@yahoo.com 


\title{
تعادل در ناشنوايان: مطالعه مرورى نظاممند
}

\author{
على اصغر نورسته'، “ حامد زارعى' \\ ا - كروه آسيبشناسى ورزشى وحركات اصلاحي، دانشكده تربيتبلني، دانشكاه كيلان، رشت، ايران.
}

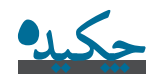

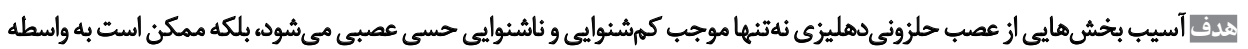

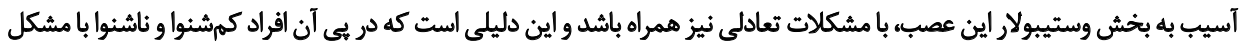

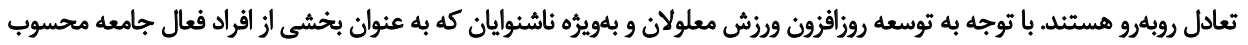

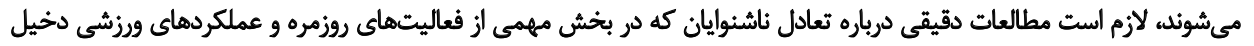

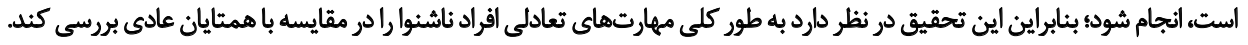

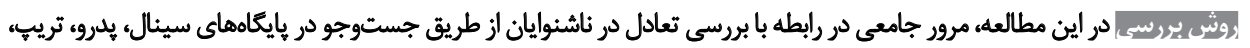

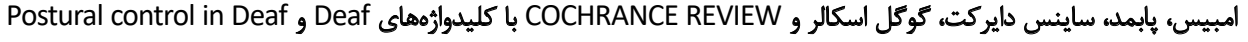
Hearing Loss، Deafness، Balance، Balance in Deaf

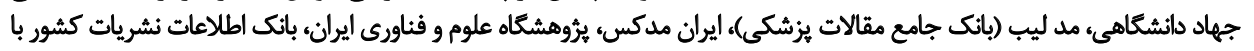

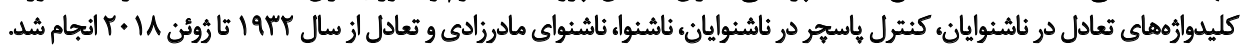

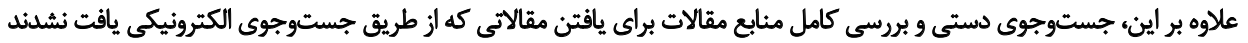

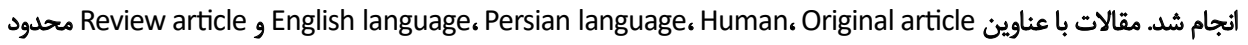

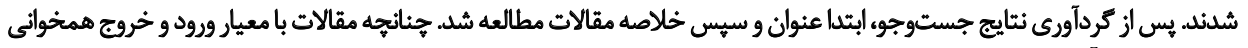

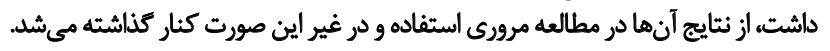

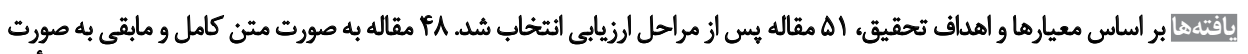

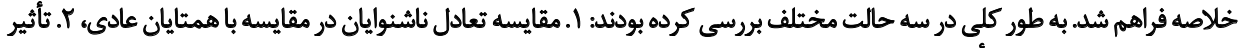

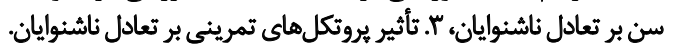

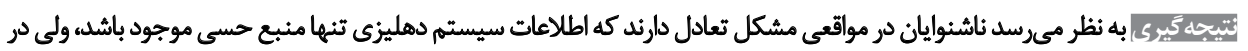

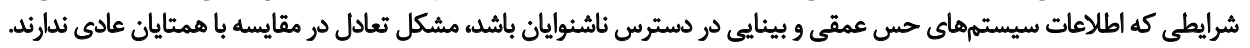

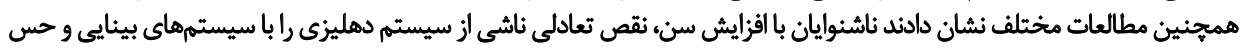

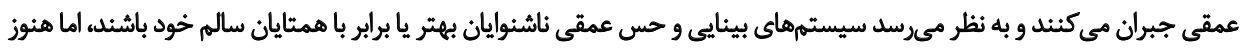

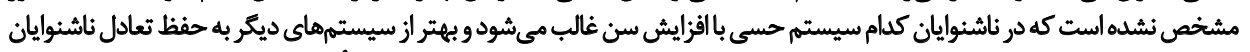

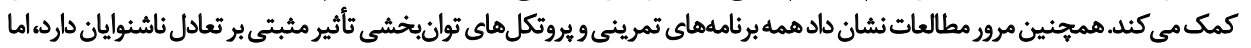

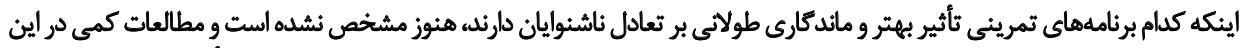

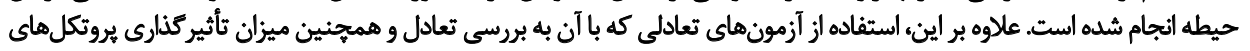

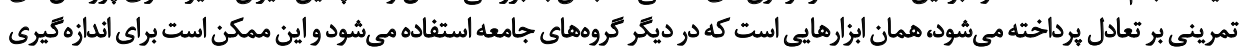

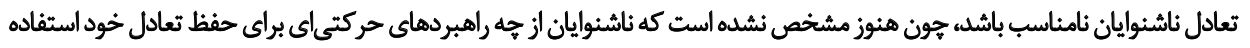

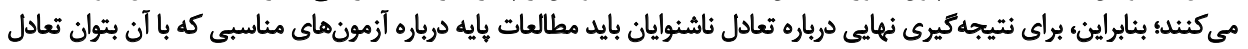

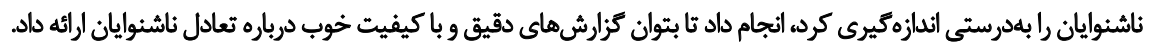

تاريخ دريافت: ץ. آذر Vqrا

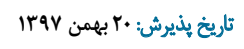

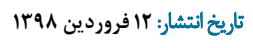

كليدوارٔهها:

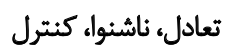

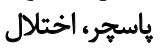

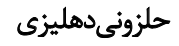




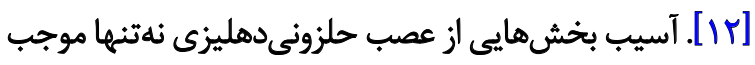

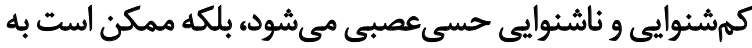

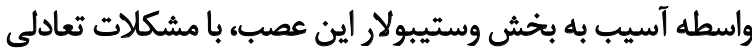

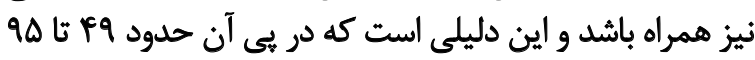

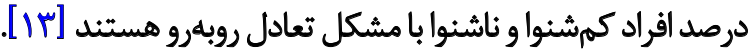

با توجه به مرور مطالعات كذشته درباره تعادل ناشنوايان،

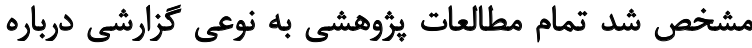

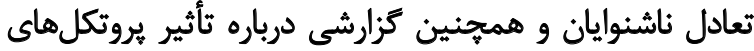

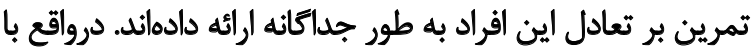

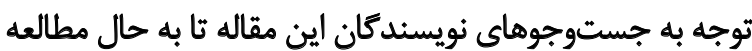

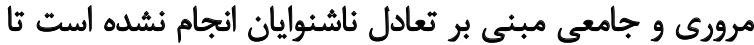

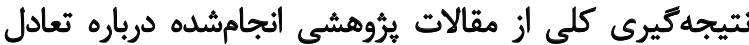

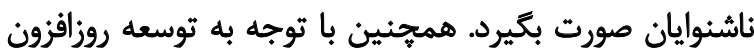

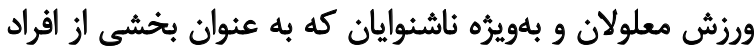

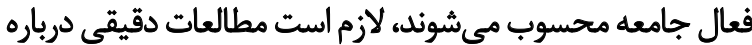

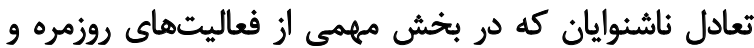

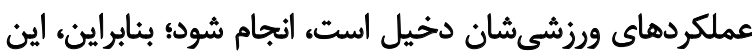

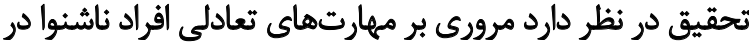

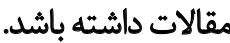

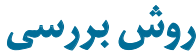

در اين مطالعه، مرور جامعى در رابطه با بررسى تعادل در

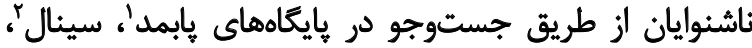

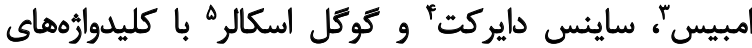
Deaf، Postural control in Deaf Hearing Loss، Deafفess، Balance، Balance in Deaf

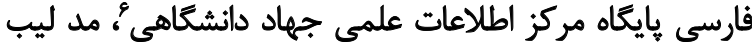

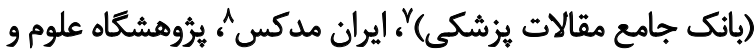

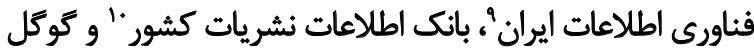

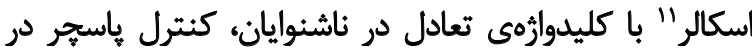

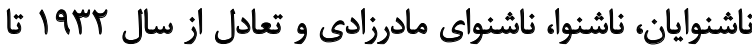

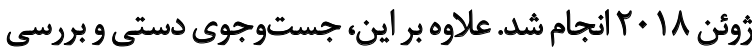

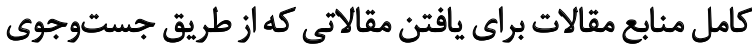
الكترونيكى يافت نشدند انجام شد.

مقالات با عناوين Persian،

1. PubMed

2. CINAHL

3. EMBASE

4. Science Direct

5. Google Scholar

6. SID

7. MedLib

8. IranMedex

9. IRANDOC

10. MAGIRAN

11. Google Scholar
مقلمه

در سالهاي اول زندگى كودكان، مهارتهاى حركتى مانند

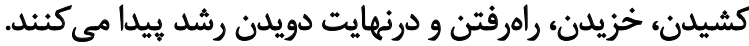

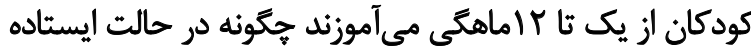

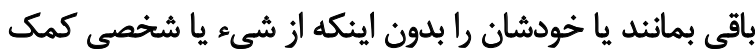

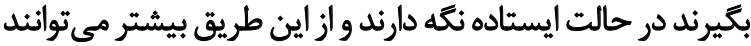

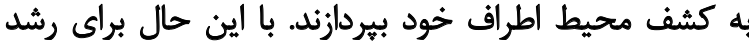

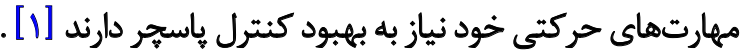
محققان، كنترل هاسجحر بلن را شامل كنترل موقعيت بلدن

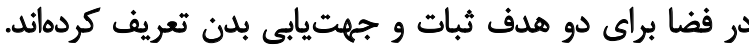

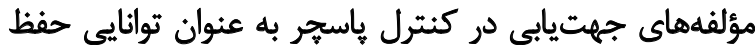

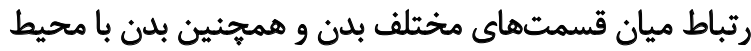

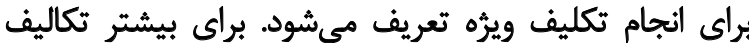

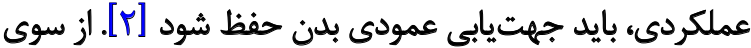

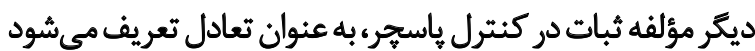

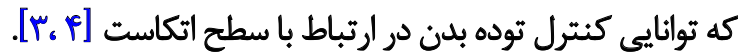
كنترل پاسجر يا حفظ تعادل نيازمند تعامل بيجيديده ميان

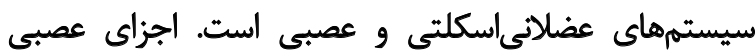

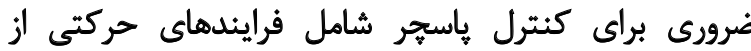

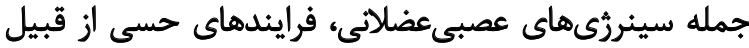

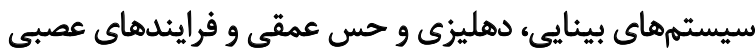

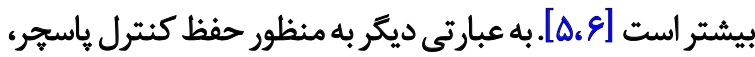

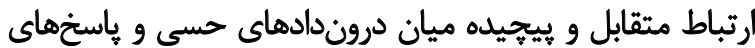

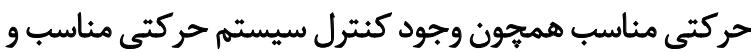

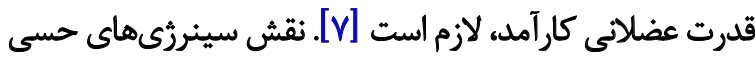
نيز در حفظ تعادل وكنترل ياسجر، در مطالعات مختلف مشخص

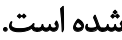

كيرندهاي وستيبولار بادريافت ورودى هاى مرتبط با موقعيت

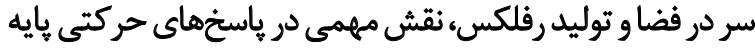

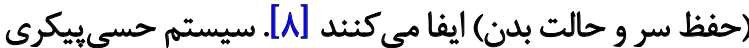

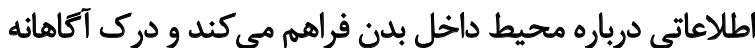

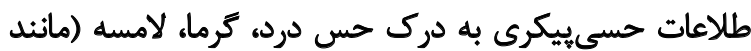

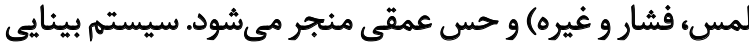

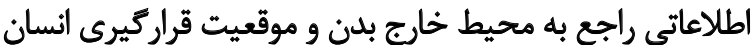

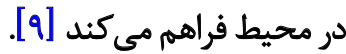

شنوايي يكى از مهمترين عوامل برقرارى ارتباط با ديكران

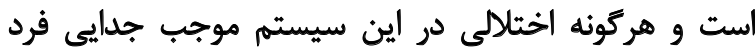

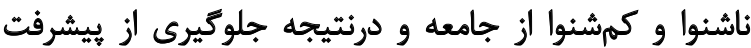

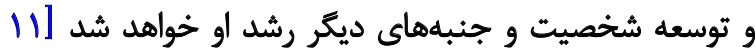

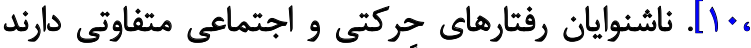

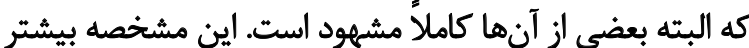

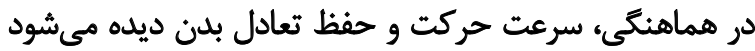




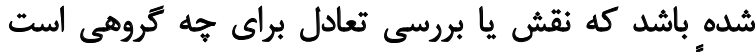

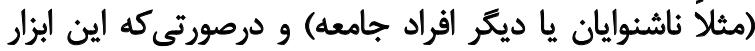

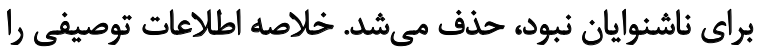

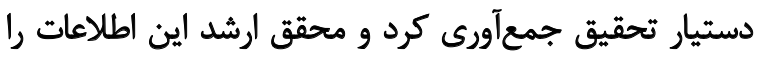

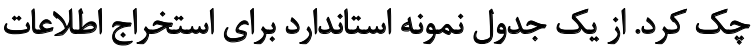
جامعه هدف، نقش تعادل در ناشنوايان و نتايج آنها استفاده شد شدات الندات (جدول شماره (1).

معيار خروج از تحقيق شامل مطالعاتى بود كه مربوط به به به

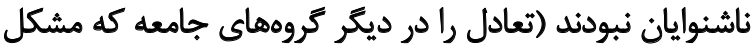

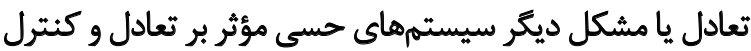
هياسجر دارند، بررسى كرده بودند).

يافتهنها

فرايند انتخاب مطالعات در تصوير شماره ا نشان داده شده

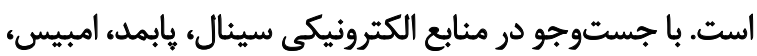

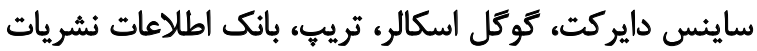
كشور، ايران مدكس، يدرو، يروهشكاه علوم و فناورى اطلاعات إنات
Review article , language،human، Original article محدود شدند. يّ از تردآورى نتايج جستوجو، ابتدا عنوان و و

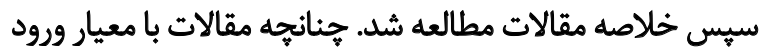

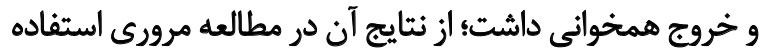

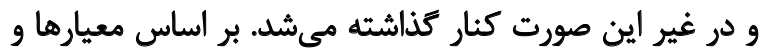

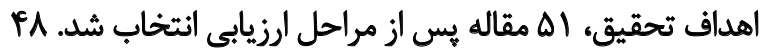

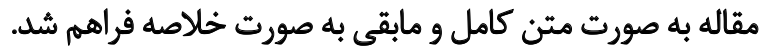
انتخاب تحقيق: در مرحله اول، غربالكرى عنوان و جـيدها

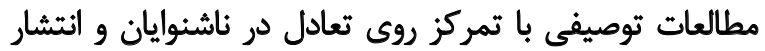

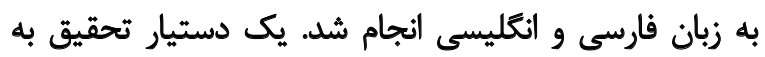

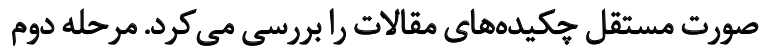

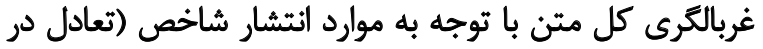

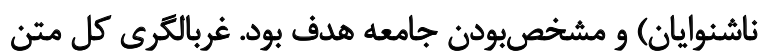

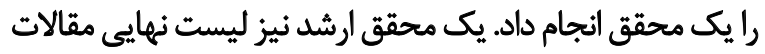
انتخابشده رابراي اطمينان از اينكه تمام مقالات با هدف تحفي نحقيق

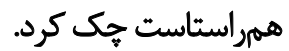

منظور از مشخصبودن جامعه هدف اين بود كه بايد بيان

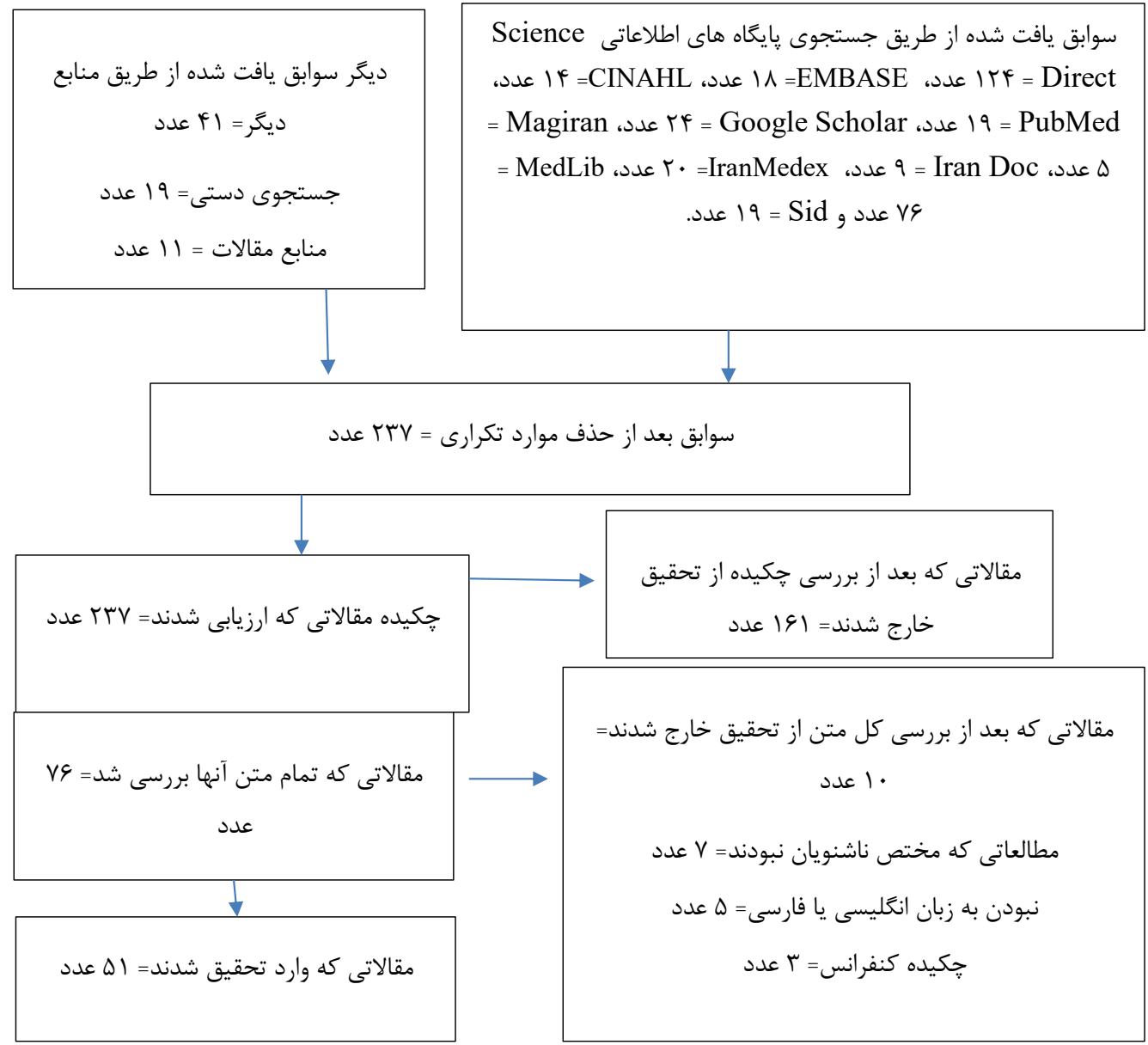


تعادل" ' مطالعه شدند. در دو وضعيت حسى (سطح ثابت و سطح

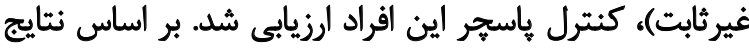

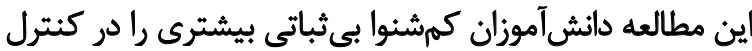

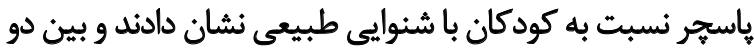

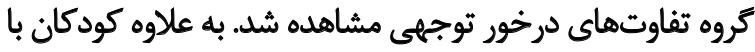

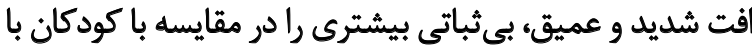

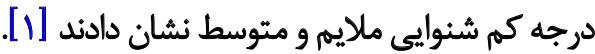
ملو" و همكاران (1) (Y) در كشور برزيل، موقعيت وامتداد سر

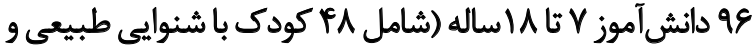

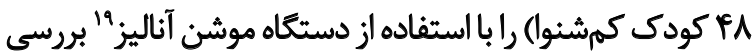

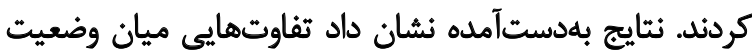

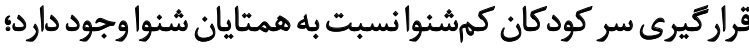

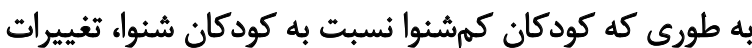

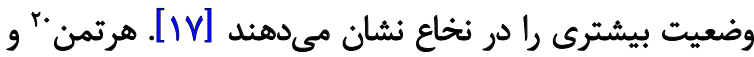

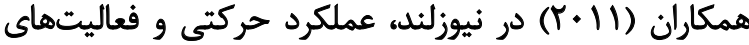

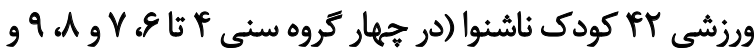

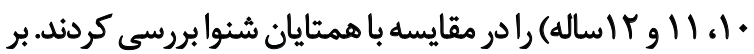

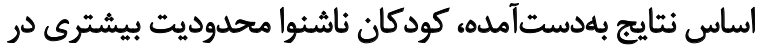

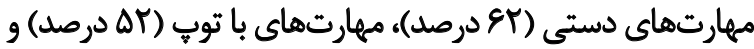

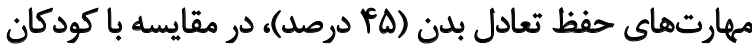

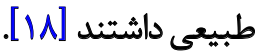

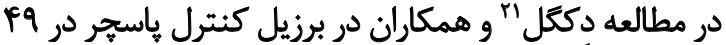

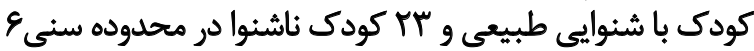

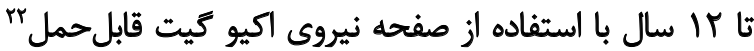

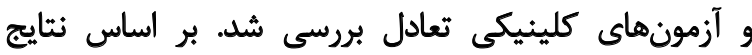

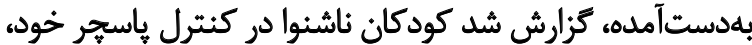

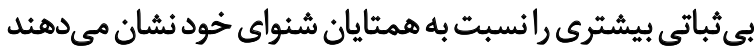

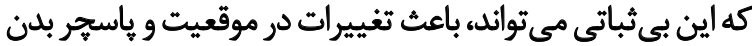

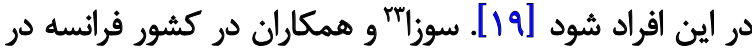

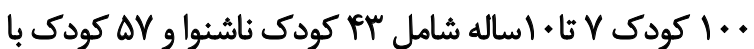

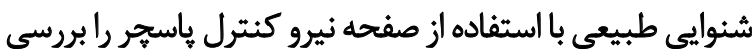

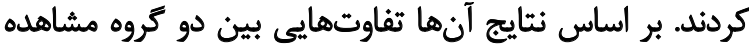

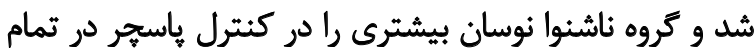

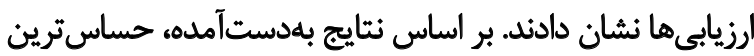

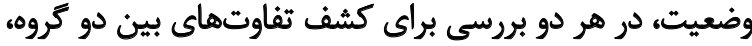

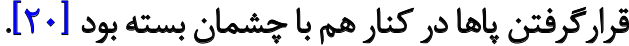
همجئين تحقيقات داخلى نيز كاهش تعادل در كودكان ناشنوا

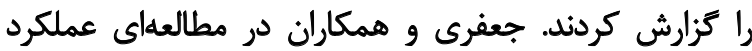

17. Balance Error Scoring System test (BESS)

18. Melo

19. Motion Analysis

20. Hartman

21. De Kegel

22. AccuGait Portable Forceplate

23. Sousa
ايران، مد ليب (بانك جامع مقالات يزشكى)، هايكاه مركز

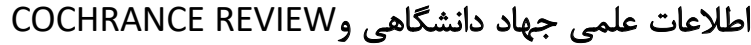

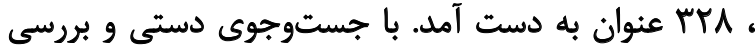

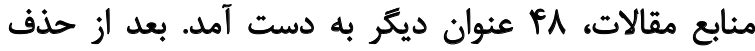

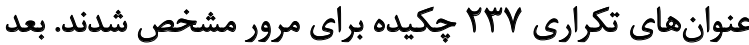

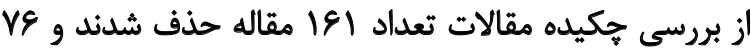

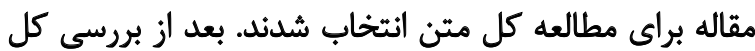

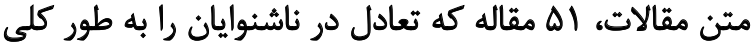

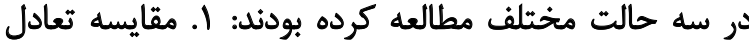

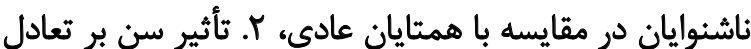

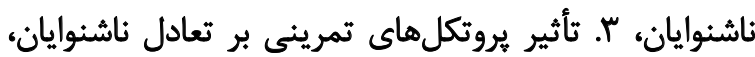

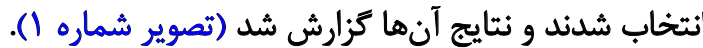
نتايج حاصل از مطالعاتى كه تعادل ناشنوايان رادر مقايسه با باديا همتايان عادى بررسى كردهانده به شرح زير است:

لانك" يكى از اولين كسانى بود كه در نيويورك، مهارتهاى

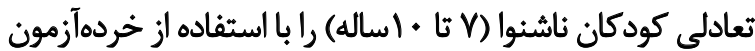

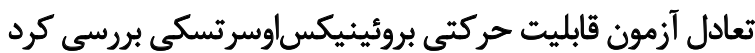

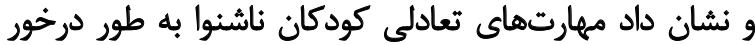

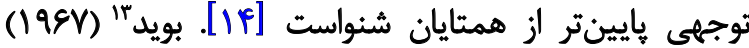

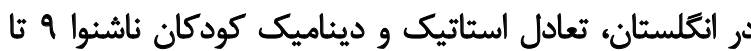

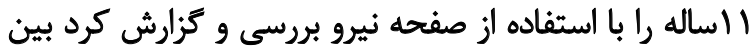

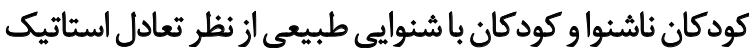

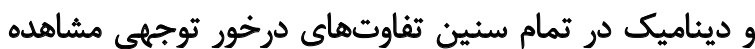

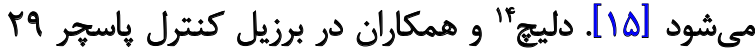

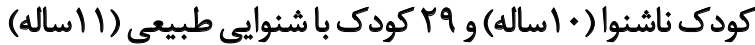

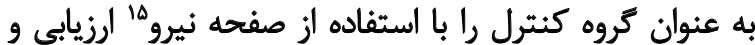

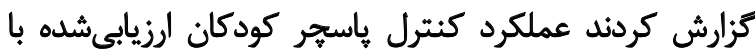

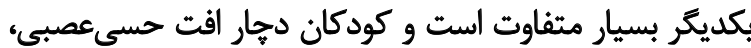

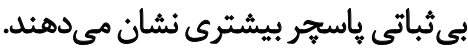

همجنين تزارش كردند در ارزيابى سطح غيرثابت، بين دو تروه

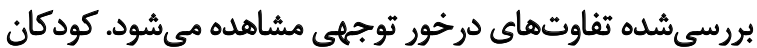

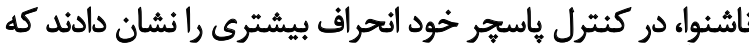

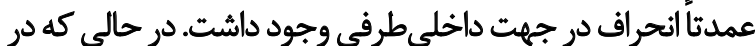

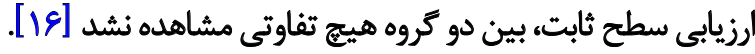

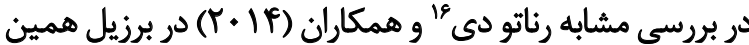

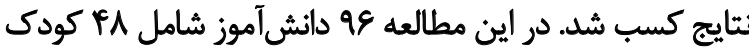

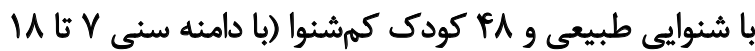

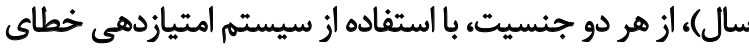

12. Long

13. Boyd

14. Derlich

15. Force platform

16. Renato de 


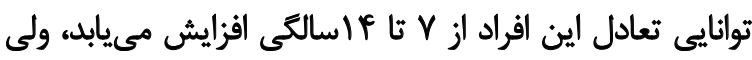

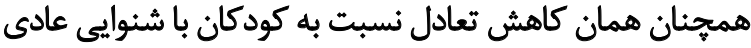

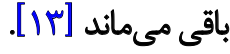

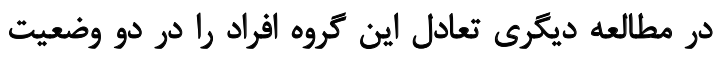

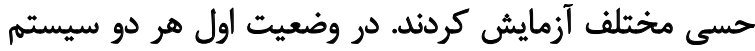

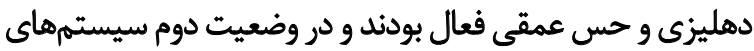

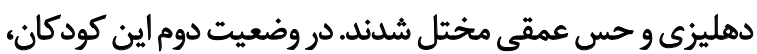

كاهش تعادل بيش از حد را مشاهده كردئد [بr.

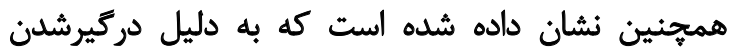

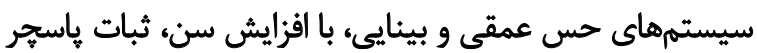

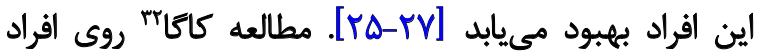

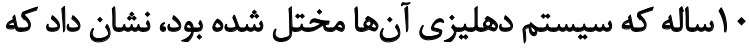

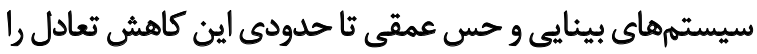

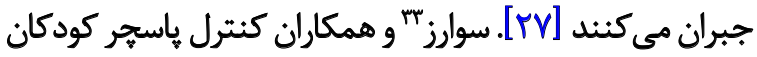

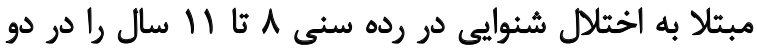

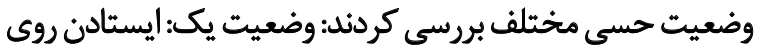

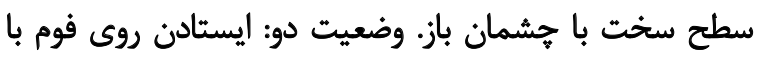

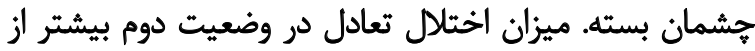

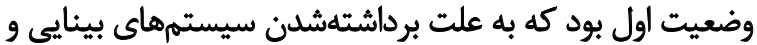

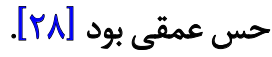

در مطالعهاى كه مايلهى "و همكارانش در سال 9 ه +. ب درباره

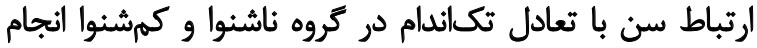

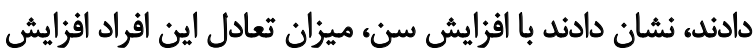

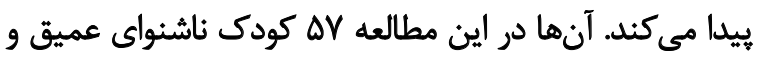

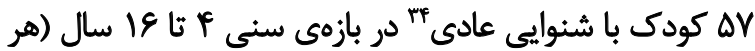

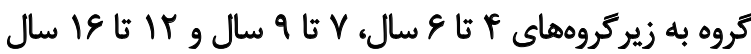

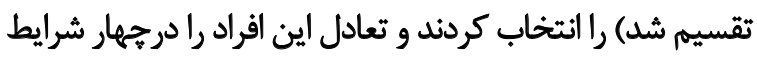

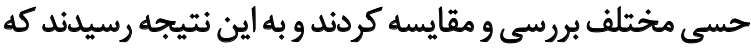

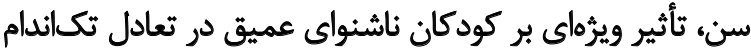

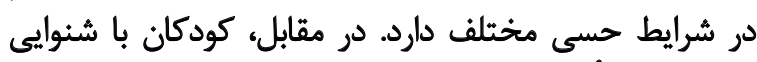

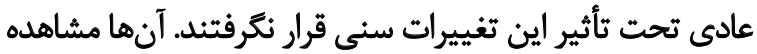

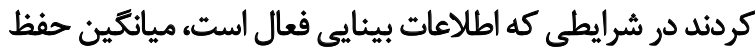

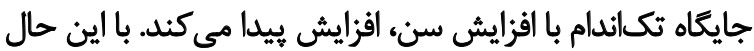

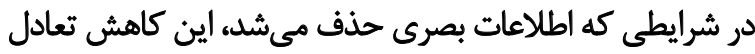
همجنان برقرار مي ماند.

اين نتايج نشان مى دهد ثبات باسجر كودكان ناشنواى عميق بإنيا

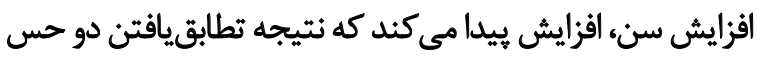

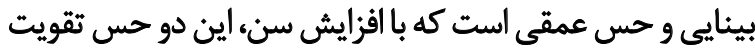

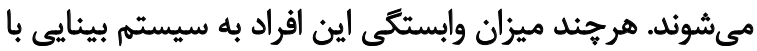

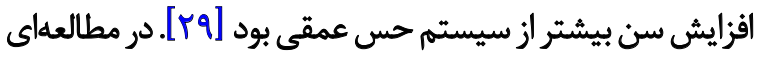

تعادلى ايستاو يويا در كودكان كمشنواي شديد تاعميق مادرزاد

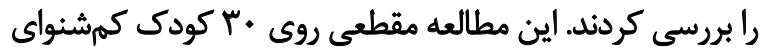

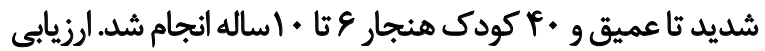

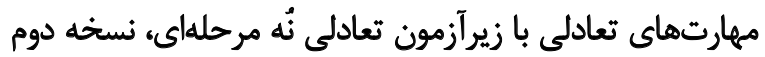

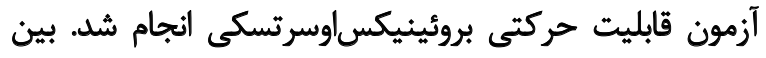

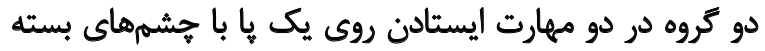

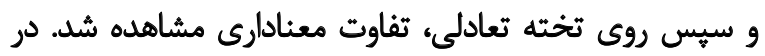

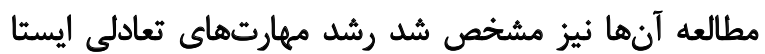

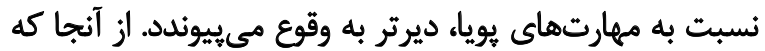

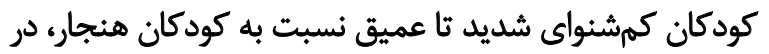

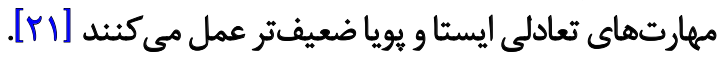

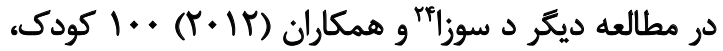

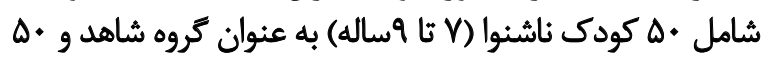

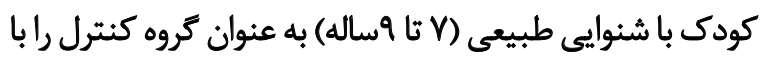

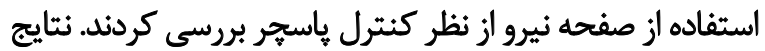

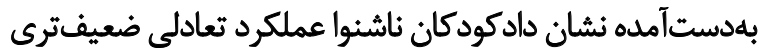

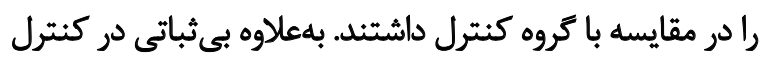

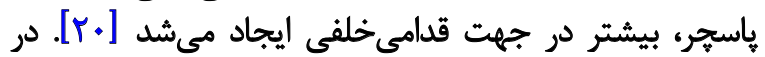

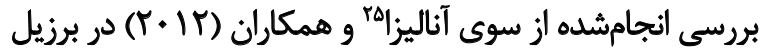

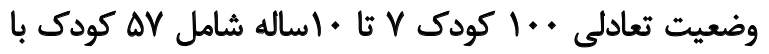

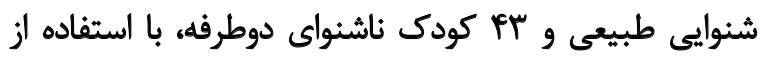
صفحه نيرو بررسى شد. بر اساس نتايج اين مطالعه كودكان ناشئوان

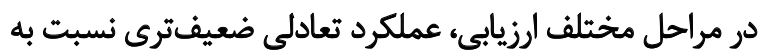

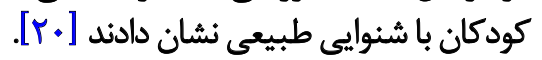

نتايج حاصل از مطالعاتى كه به تطبيق كنترل ياسجر ناشنوايان با افزايش سن برداختهاند، يه شرح زير است:

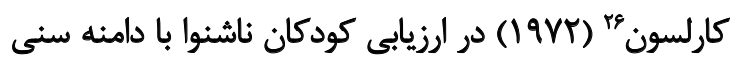

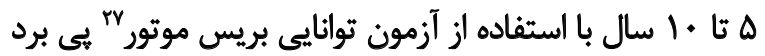

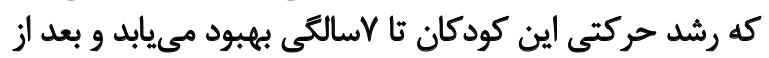

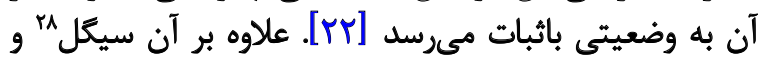

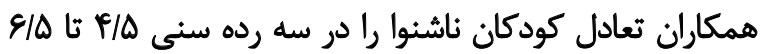

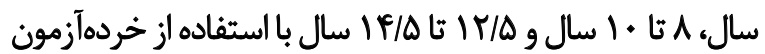

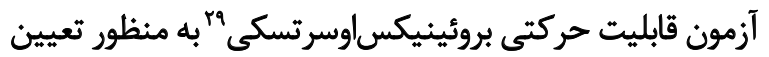

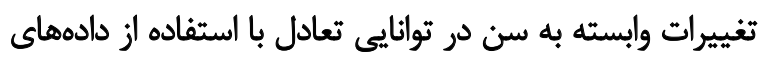

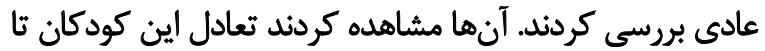

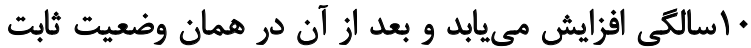

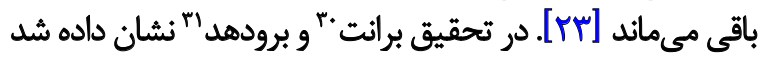

24. De Sousa

25. Aneliza

26. Carlson

27. Brace- Motor

28. Siegel

29. Bruininks-Oseretsky Test of Motor Proficiency

30. Brunt

31. Broadhead 


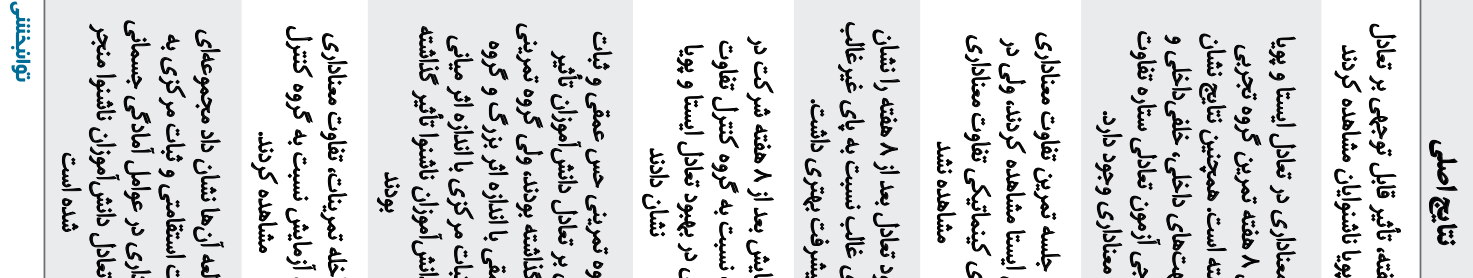

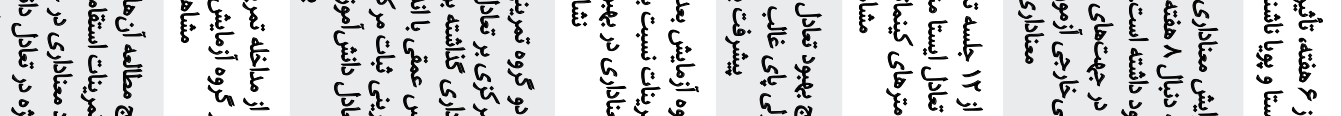

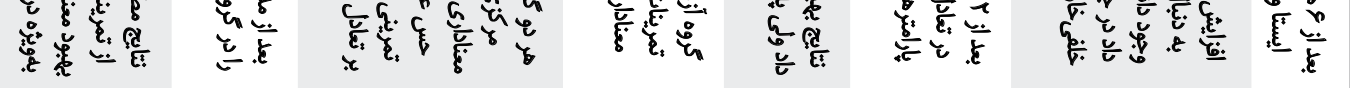

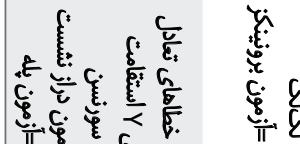

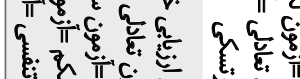

Git

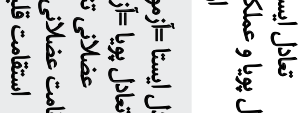

$\underline{\underline{\varepsilon}}$

द.

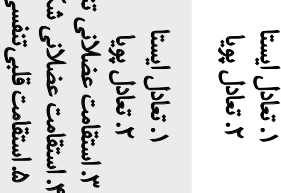

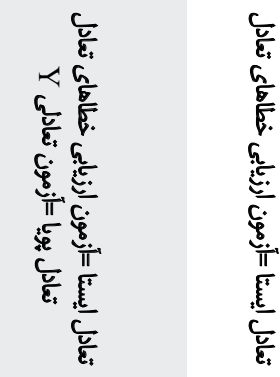

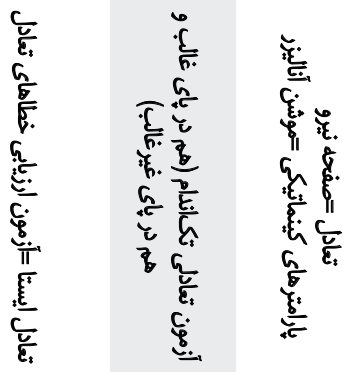

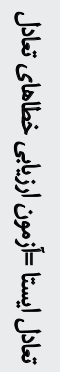

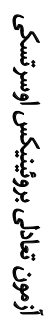

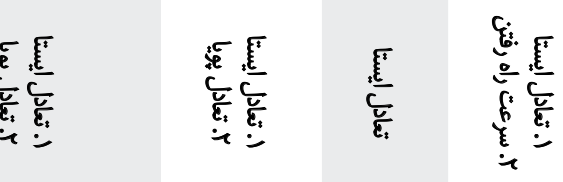

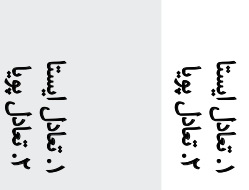

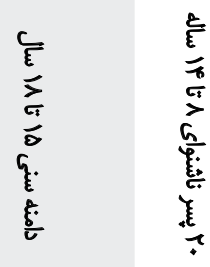

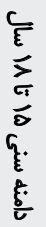

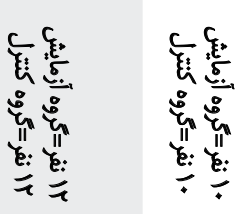

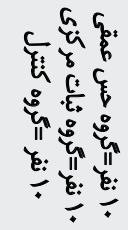

要:

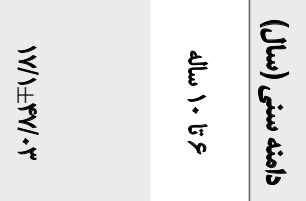

है है

高

急蓄

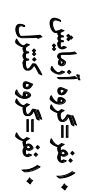

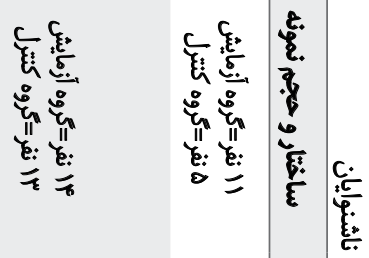

$\hat{\Xi}$

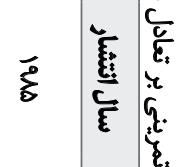

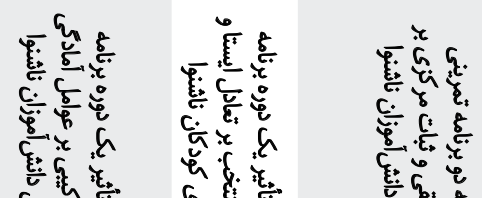

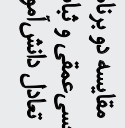

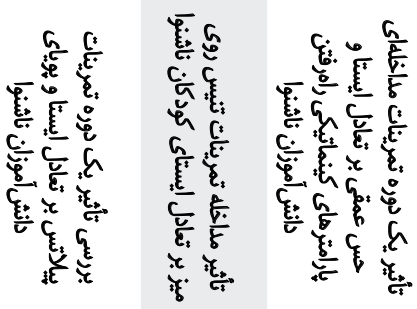

篦

管

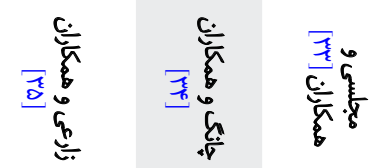

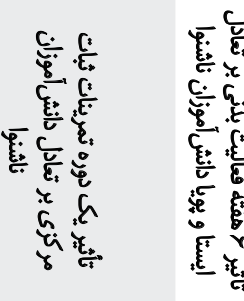

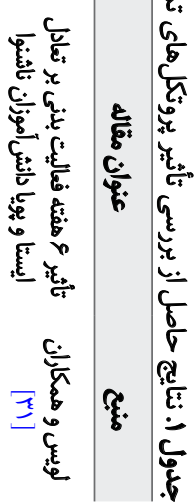


عصبىمركزى را قادر ميى كند كه در هر لحظه از وضعيت بلدن در

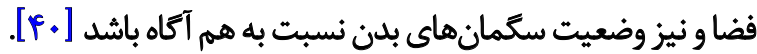
بر اساس مطالعات انجامشده، كاهش فعاليت سيستم دهليزى

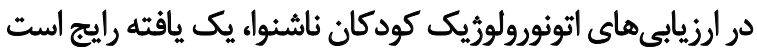

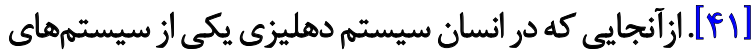

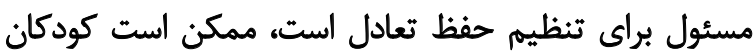

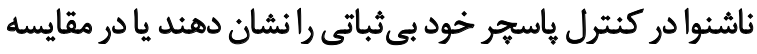

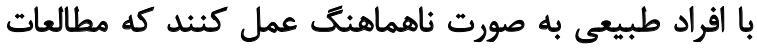

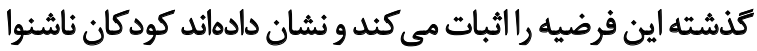

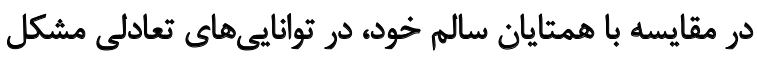

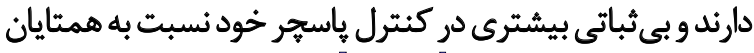

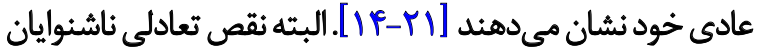
را مى توان از ديدكاه ديكر مشاهده و بردسي كرد.

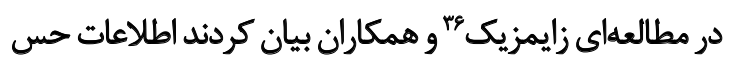

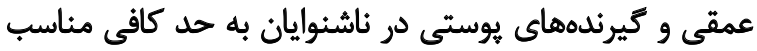

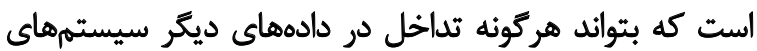

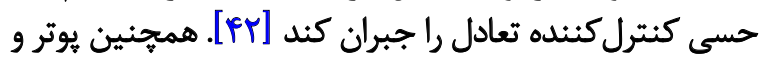

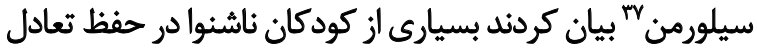

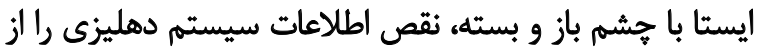

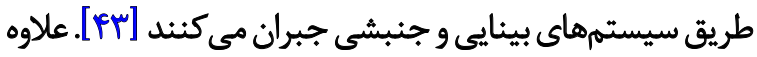

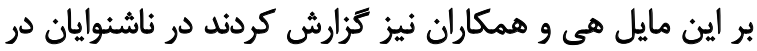

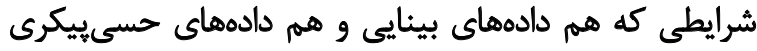

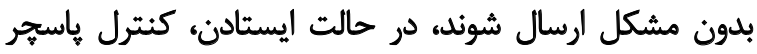

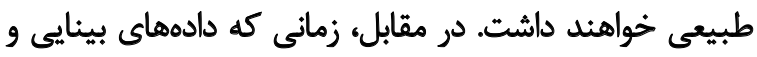

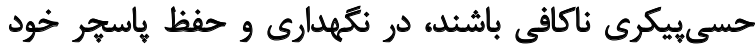

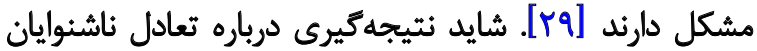

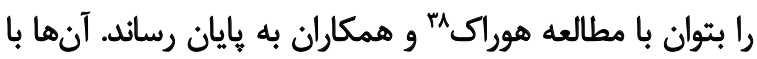

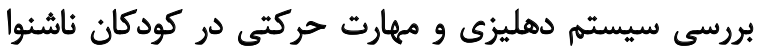

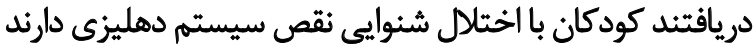

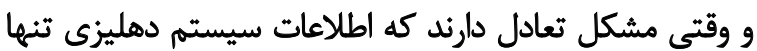

منبع حسى موجود باشد [Ff]

$$
\text { ثأثير سن بر تعادل ناششنوايان }
$$

بر اساس ديدكاه فيزيولوريكى، قبل از اينكه عملكرد كنترل

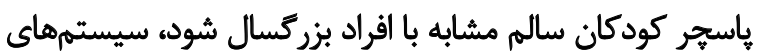

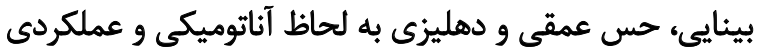

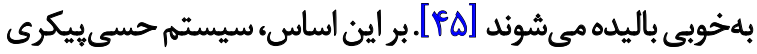

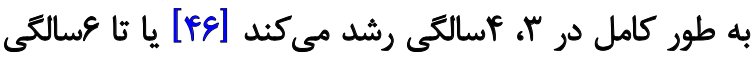

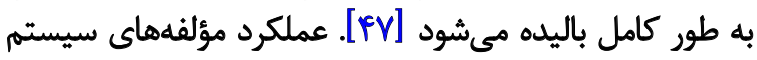
دهليزى (شامل هجارى نيمدايره، اندامهاى اوتوليتي و ميزان

36. Szymczyk

37. Potter \& Silverman

38. Horak
ديكر وليكا كويريس ها و همكاران سيستمهاى حسى دركير در

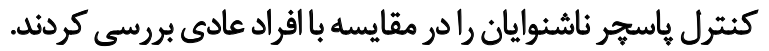

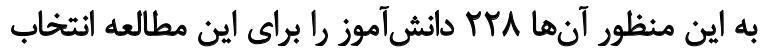

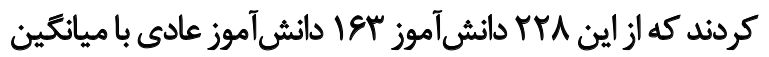

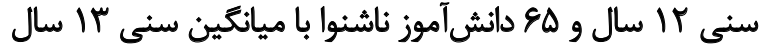

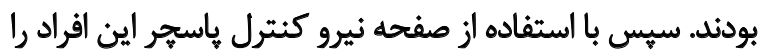

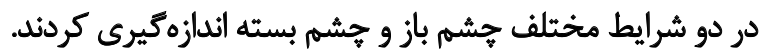

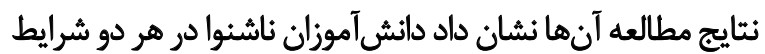

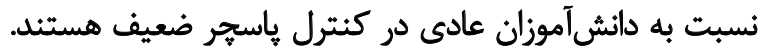

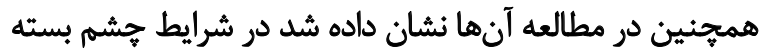

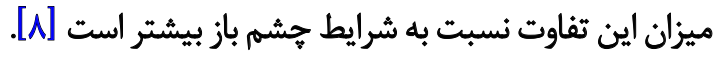
سيدى و همكاران در مطالعهاى ميزان كارايى سيستمهاى

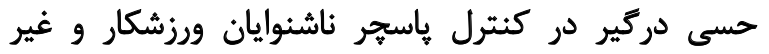

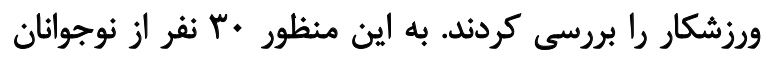

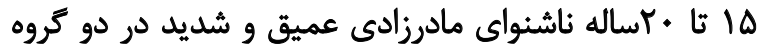

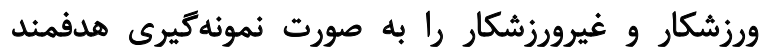

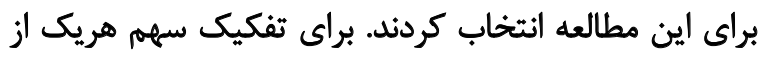

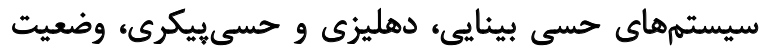

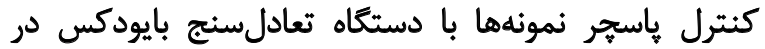

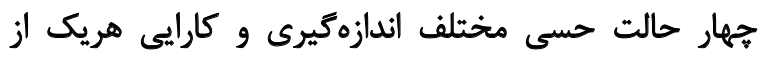

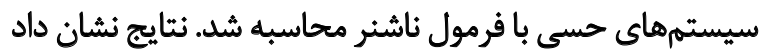

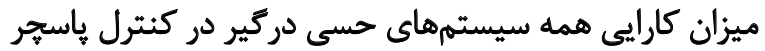

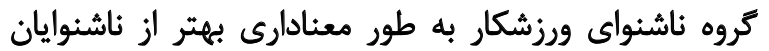

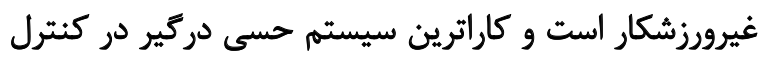

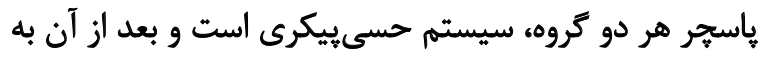

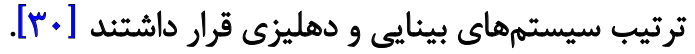

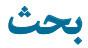
هدف از اين مطالعه بررسى تعادل در ناشنوايان بود؛ با مرور

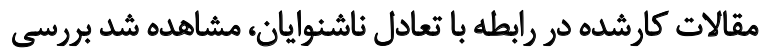

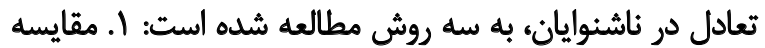

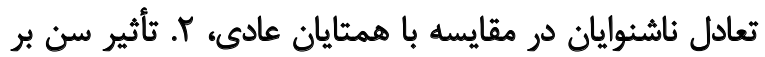

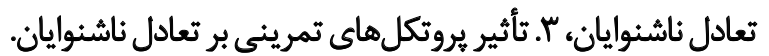

$$
\text { مقايسه تعادل ناثشئوايان در مقايسه با همثايان عادى }
$$

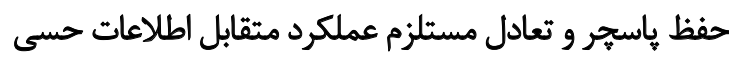

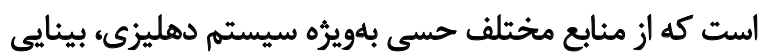

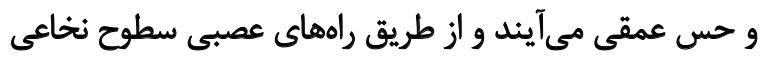

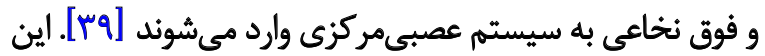

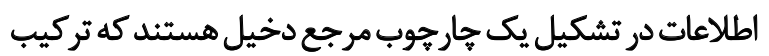

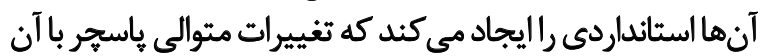
سنجيده مىشود و درواقع شماى كلى بدن رانئن امىسازد و سيستم

35. Walicka-Cupryś 


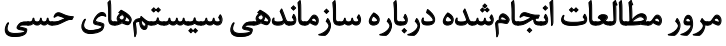

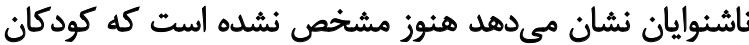

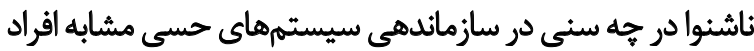

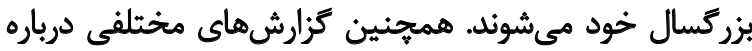

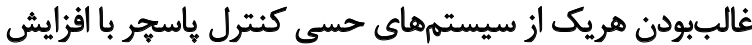
سن در افراد سالم و ناشنوا كزارش شده است.

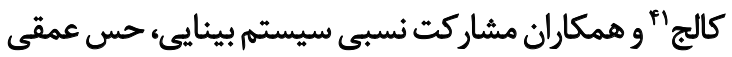

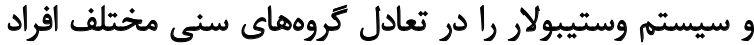

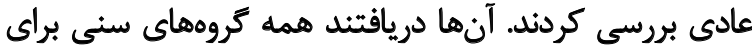

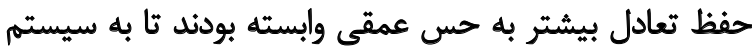

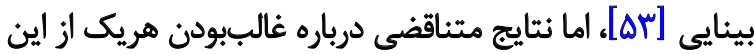

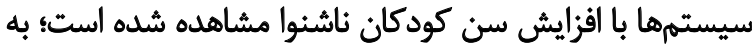

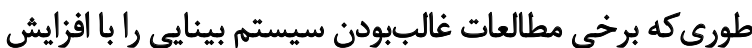

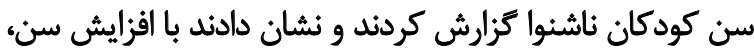

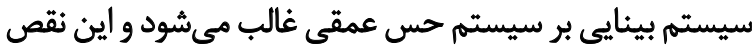

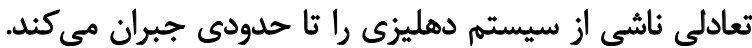

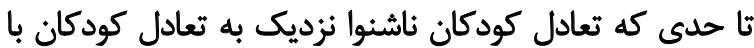
شنوايى عادى قرار بكيرد [ra] تادئ. در حالى كه برخى مطالعات دقيقاً بر عكس جمله قبلى راعنوان

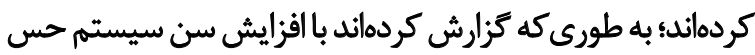

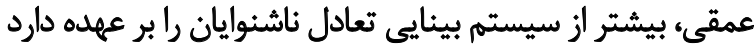

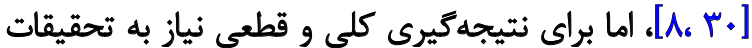

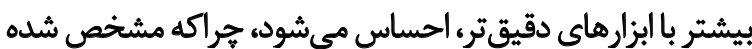

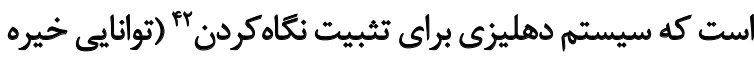

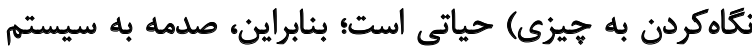

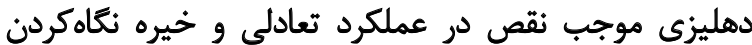

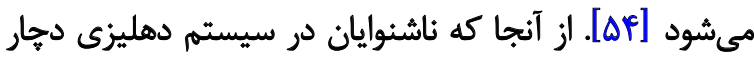
ضعف و نقص هستند و همان طور كه بيان شد اطلاعات سيستم

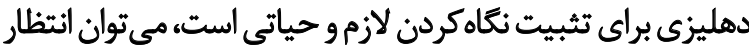

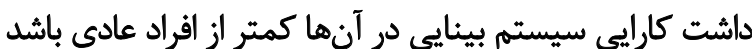

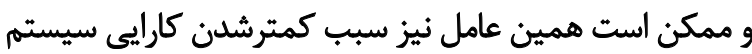
بينايي نسبت به سيستم حسي عييكرى در ناشنوايان باشد.

\section{تأثير يروتكلهاي تمرينى بر تعادل ناشينوايان}

مطالعات عنوان كردهاند كه بروتكل هاى تمرينى و برنامههاى تواي

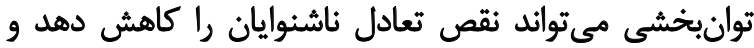

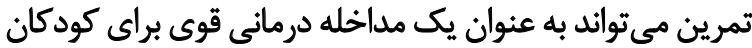

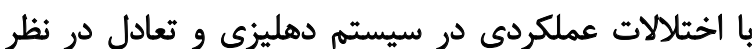

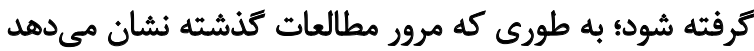

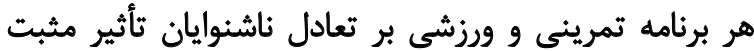

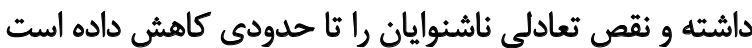

41. Colledge

42. Gaze stabilization
ميليندارشدن عصب دهليزى) در زمان تولد مشابه با افراد

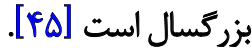

از سوى ديكر ميزان باليدكى سيستم بينايى بسيار متغير است؛

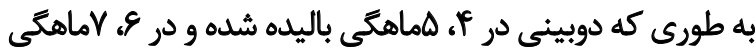

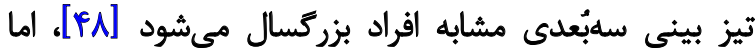

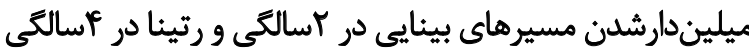

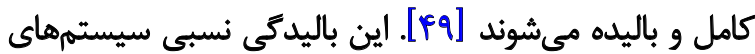

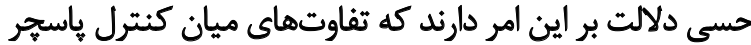

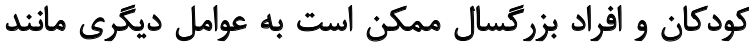

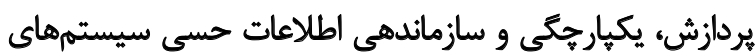

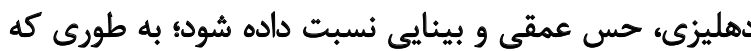

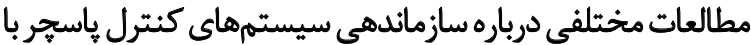
افزايش سن انجام و نتايج مختلفى نيز كزارش شارئ شده است.

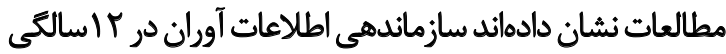

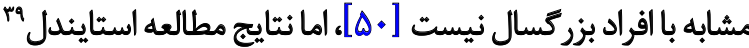

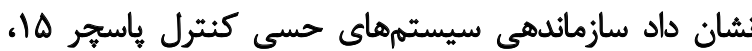

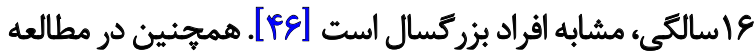

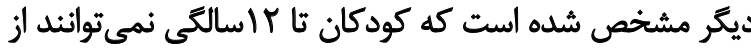

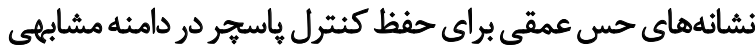

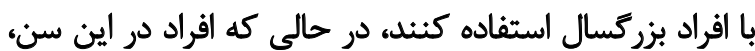

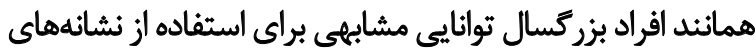

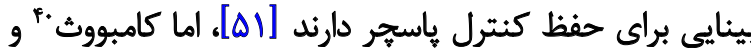

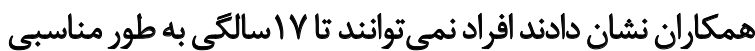

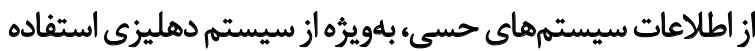

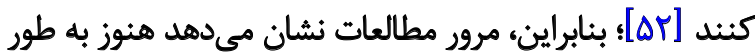

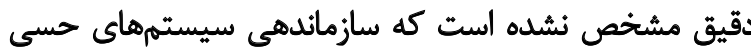

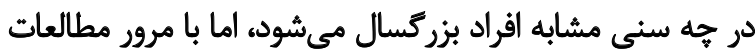

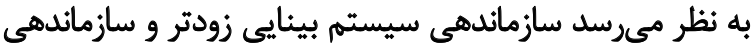

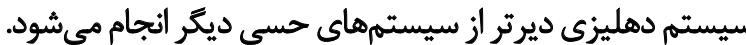

نتايج مطالعات در كروه ناشنوايان به كونهاى متفاوتتر از

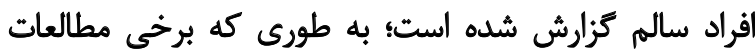

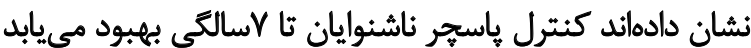

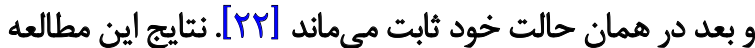

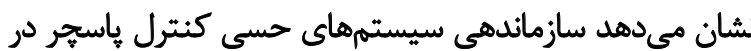

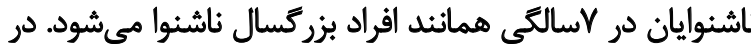

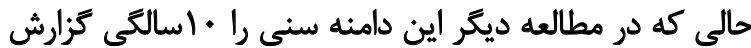

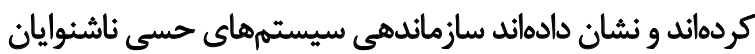

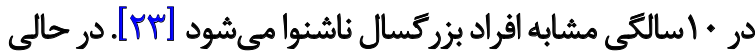

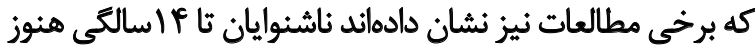

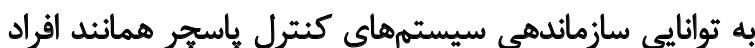
بزركسال خود نرسيدهاند [11]]

39. Steind

40. Cumberworth 
شايد اين عوامل يكى از عواملى باشد كه باعث ضعف در تعادل

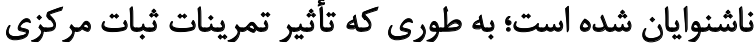

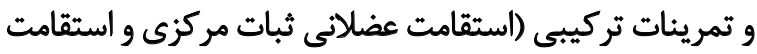

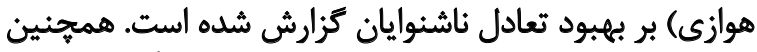

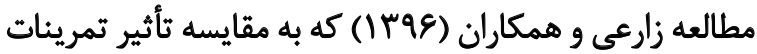

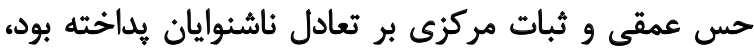

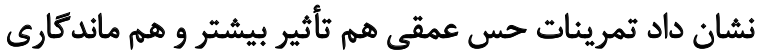

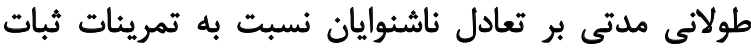

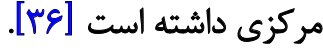

همجنين مشاركتنداشتن در فعاليتهاى ورزشى، به داليل

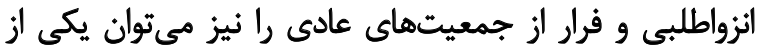

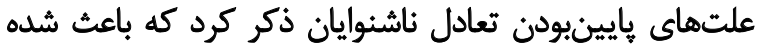

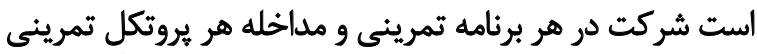

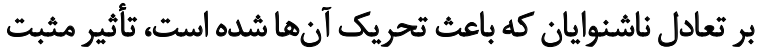

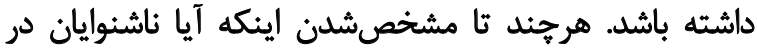

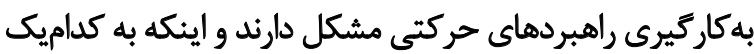

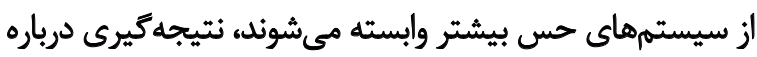

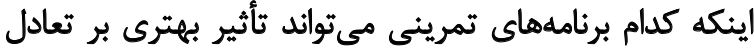
ناشنوايان داشته باشد، سخت است

اين مطالعه مرورى روش جستوجوى نظامهند داشت،

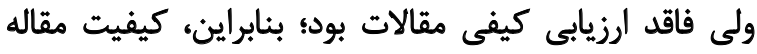

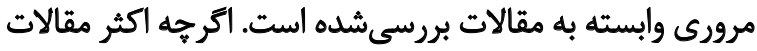

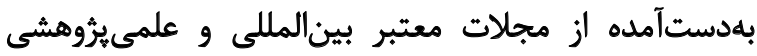

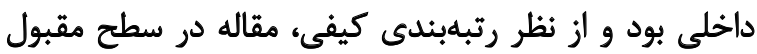

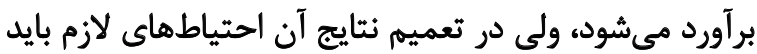

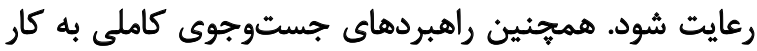

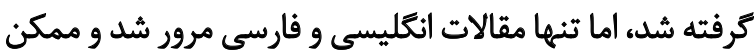

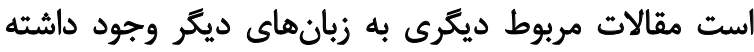

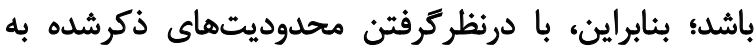

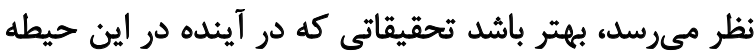
صورت مى ميرد به بررسى كيفى مقالات بيردازئد.

تثيجنيرى

به نظر مىرسد ناشنوايان در مواقعى مشكل تعادل دارند كه

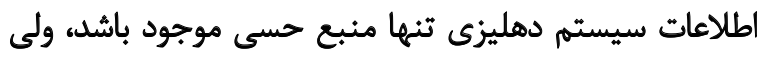

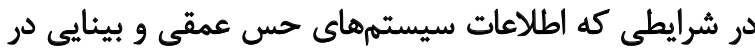

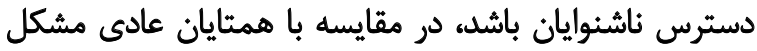

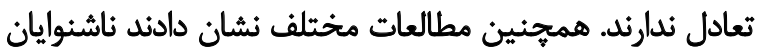

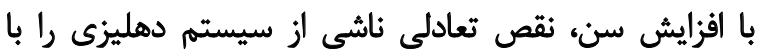

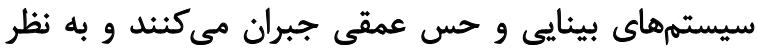

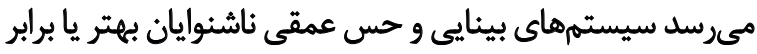

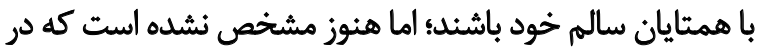
ناشنوايان كدام سيستم حسى با بافزايش سن غالب مى بشود و بهتر

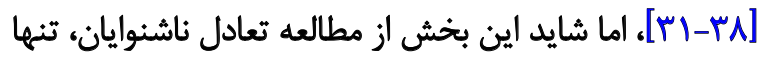

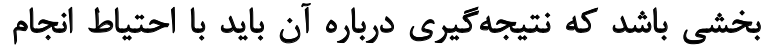

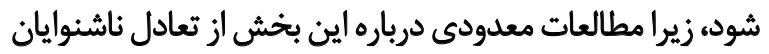

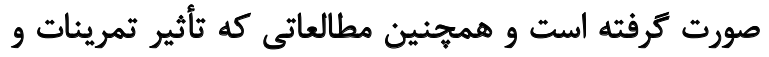

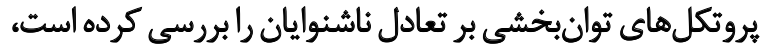

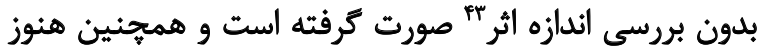

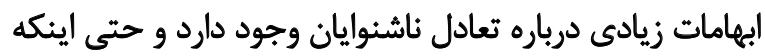

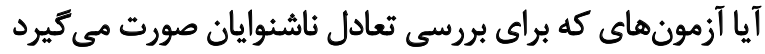

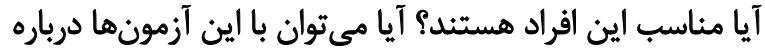

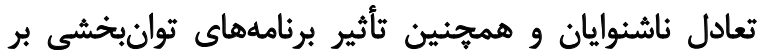

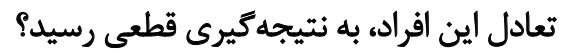
براى مثال ذكر شده است سيستم وستيبولار در ايجاد راهبرد

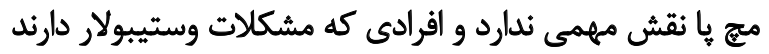

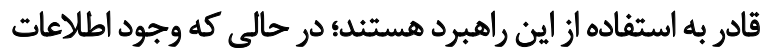

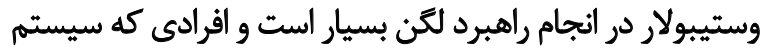

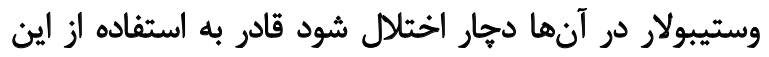

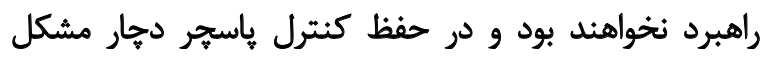

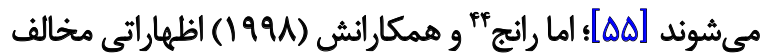

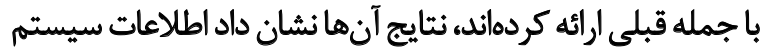

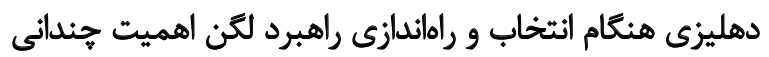

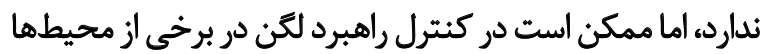

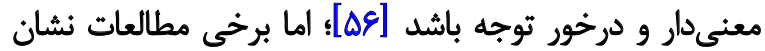

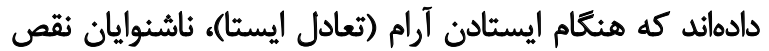

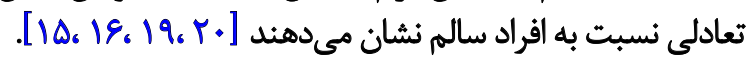

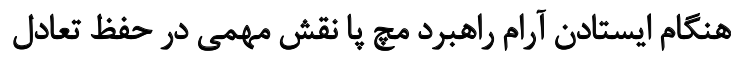

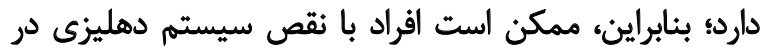

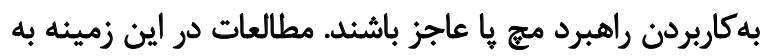

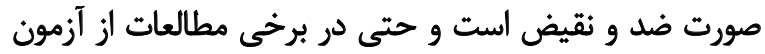

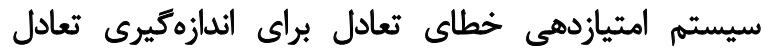

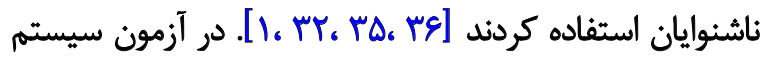

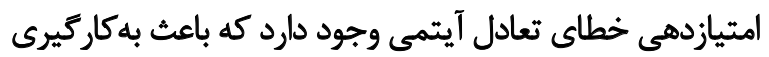

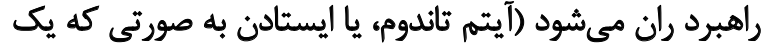

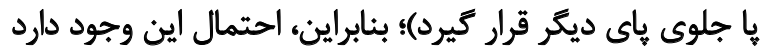

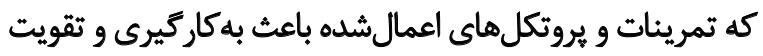

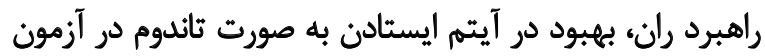

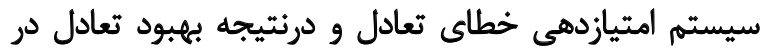

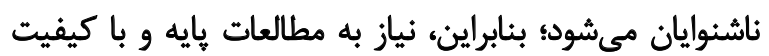
مناسب درباره تعادل ناشنوايان هست.

در مطالعاتى ذكر شده است كه ناشنوايان سطوح آمادكى

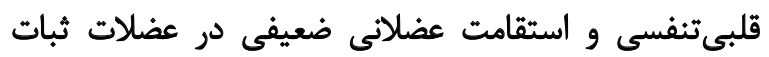

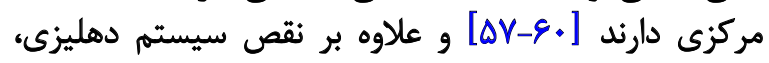


از سيستمهاى ديكر به حفظ تعادل ناشنوايان كمك مى كند. همجينين مرور مطالعات نشان داد همه برنامههاي تمرينى و

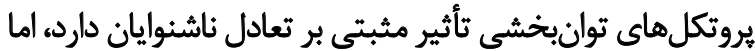

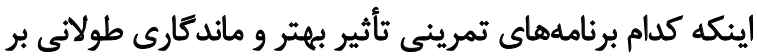

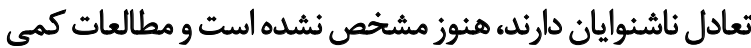

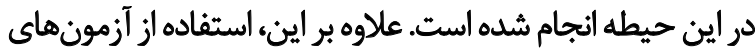

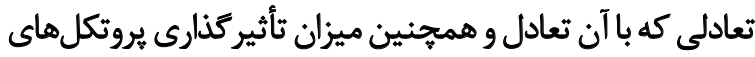

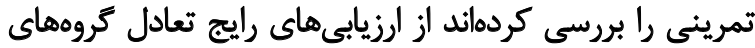

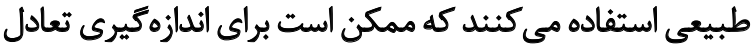

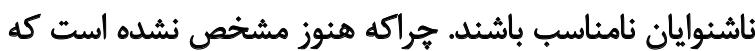

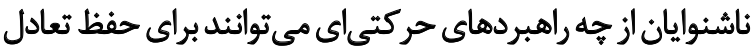

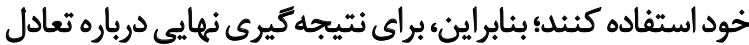

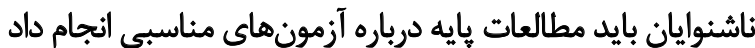

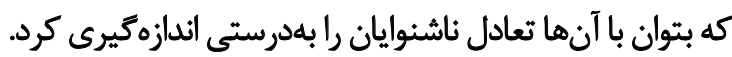

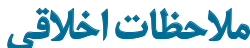
ييروى أز اصول اخلاق يُوهش با توجه به اينكه مقاله از نوع مرورى است، اصول اخلاقي وجود

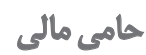

اين مقاله برگرفته از هاياننامه كارشناسى ارشد حامد زارعى در

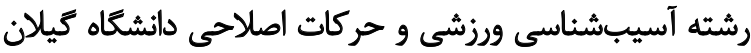

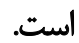

$$
\text { مشاركت نويسند كَان }
$$

همه نويسندكان در آمادهسازى اين مقاله مشاركت داشتهاند.

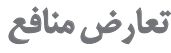

بنابر اظهار نويسندكان، اين مقاله تعارض مثافع ندارد. 


\section{References}

[1] Melo RDS, Lemos A, Macky CFdST, Raposo MCF, Ferraz KM. Postural control assessment in students with normal hearing and sensorineural hearing loss. Brazilian Journal of Otorhinolaryngology. 2015; 81(4):431-8. [DOI:10.1016/j. bjorl.2014.08.014] [PMID]

[2] Khodabakhshi $M$, Ebrahimi-A'tri A, Hashemi-Javaheri SAA, Khan-Zadeh R, Zandi M. [The effect of 5 weeks proprioceptive training on basketball player's dynamic balance with aspirin for chronic ankle (Persian)]. Archives of Rehabilitation. 2014 15(3):44-51

[3] Shumway-Cook A, Woollacott MH. Motor control: Translating research into clinical practice. Philadelphia, Pennsylvania: Lippincott Williams \& Wilkins; 2007. [PMCID]

[4] Farhadian M, Bozorgi J, Asghar A, Ahmadi Fakhreh M, Morovati Z, Qafarizadeh F. Effect of gait retraining on balance, activities of daily living, quality of life and depression in stroke patients. Iranian Rehabilitation Journal. 2015; 13(4):116-9.

[5] Kamalian Lari S, Haghgoo HA, Farzad M, Hosseinzadeh S. Investigation of the Validity and Reliability of Balance Evaluation Systems Test (BESTest) in assessment of balance disorders in People with Multiple Sclerosis. Archives of Rehabilitation. 2018; 18(4):288-95. [DOI:10.21859/jrehab.18.4.3]

[6] Pourkhani T, Norasteh AA, Shamsi A. [Effect of ankle taping and fatigue on dynamic stability in athletes with and without chronic ankle instability (Persian)]. Archives of Rehabilitation. 2017; 18(2):110-21. [DOI:10.21859/jrehab-1802108]

[7] Da Silva RA, Bilodeau M, Parreira RB, Teixeira DC, Amorim CF. Age-related differences in time-limit performance and force platform-based balance measures during one-leg stance. Journal of Electromyography and Kinesiology. 2013; 23(3):634-9. [DOI:10.1016/j.jelekin.2013.01.008] [PMID]

[8] Walicka-Cupryś K, Przygoda $九$, Czenczek E, Truszczyńska A, Drzał-Grabiec J, Zbigniew T, et al. Balance assessment in hearing-impaired children. Research in Developmental Disabilities. 2014; 35(11):2728-34. [DOI:10.1016/j.ridd.2014.07.008] [PMID]

[9] Riemann BL, Lephart SM. The sensorimotor system, part I: The physiologic basis of functional joint stability. Journal of Athletic Training. 2002; 37(1):71-9. [PMID] [PMCID]

[10] Khalifeh Loo S, Mobaraki H, Kamali M, Jafari Z. [Research paper: designing and determining validity and reliability of the questionnaire for parents satisfaction with the services provided for children with hearing loss (Persian)]. Archives of Rehabilitation. 2016; 17(3):244-51. [DOI:10.21859/jrehab-1703244]

[11] Borujeni SS, Hatamizadeh N, Vameghi R, Karaskian A. Hearing loss related quality of life in adolescents with hearing loss. Iranian Rehabilitation Journal. 2015; 13(1):38-43.

[12] Cushing SL, Chia R, James AL, Papsin BC, Gordon KA. A test of static and dynamic balance function in children with cochlear implants: the vestibular Olympics. Archives of Otolaryngology-Head \& Neck Surgery. 2008; 134(1):34-8. [DOI:10.1001/ archoto.2007.16] [PMID]
[13] Brunt D, Broadhead GD. Motor proficiency traits of deaf children. Research Quarterly for Exercise and Sport. 1982; 53(3):236-8. [DOI:10.1080/02701367.1982.10609346]

[14] Long JA. Motor abilities of deaf children. New York: Columbia University; 1932.

[15] Boyd J. Comparison of motor behavior in deaf and hearing boys. American annals of the deaf. 1967; 112(4):598-605 [PMID]

[16] Derlich M, Kręcisz K, Kuczyński M. Attention demand and postural control in children with hearing deficit. Research in Developmental Disabilities. 2011; 32(5):1808-13. [DOI:10.1016/j. ridd.2011.03.009] [PMID]

[17] de Souza Melo R, da Silva PWA, da Silva LVC, da Silva Toscano CF. Postural evaluation of vertebral column in children and teenagers with hearing loss. International Archives of Otorhinolaryngology. 2011; 15(02):195-202. [DOI:10.1590/S180948722011000200012

[18] Hartman E, Houwen S, Visscher C. Motor skill performance and sports participation in deaf elementary school children. Adapted Physical Activity Quarterly. 2011; 28(2):132-45. [DOI:10.1123/apaq.28.2.132] [PMID]

[19] De Kegel A, Dhooge I, Cambier D, Baetens T, Palmans T, Van Waelvelde H. Test-retest reliability of the assessment of postural stability in typically developing children and in hearing impaired children. Gait \& Posture. 2011; 33(4):679-85 [DOI:10.1016/j.gaitpost.2011.02.024] [PMID]

[20] de Sousa AMM, de França Barros J, de Sousa Neto BM. Postural control in children with typical development and children with profound hearing loss. International Journal of General Medicine. 2012; 2012:433-9. [DOI:10.2147/IJGM.S28693] [PMID] [PMCID]

[21] Jafari Z, Malayeri S, Rezazadeh N, HajiHeydari F. Static and dynamic balance in congenital severe to profound hearingimpaired children. Audiology. 2011; 20(2):102-12.

[22] Carlson BR. Assessment of motor ability of selected deaf children in Kansas. Perceptual and Motor Skills. 1972; 34(1):3035. [DOI:10.2466/pms.1972.34.1.303] [PMID]

[23] Siegel JC, Marchetti M, Tecklin JS. Age-related balance changes in hearing-impaired children. Physical Therapy. 1991; 71(3):183-9. [DOI:10.1093/ptj/71.3.183] [PMID]

[24] Horak F, Nashner L, Diener H. Postural strategies associated with somatosensory and vestibular loss. Experimental Brain Research. 1990; 82(1):167-77. [DOI:10.1007/BF00230848] [PMID]

[25] Nashner LM. Adaptation of human movement to altered environments. Trends in Neurosciences. 1982; 5:358-61. [DOI:10.1016/0166-2236(82)90204-1]

[26] Dummer GM, Haubenstricker JL, Stewart DA. Motor skill performances of children who are deaf. Adapted Physical Activity Quarterly. 1996; 13(4):400-14. [DOI:10.1123/apaq.13.4.400]

[27] Kaga K. Vestibular compensation in infants and children with congenital and acquired vestibular loss in both ears. In- 
ternational Journal of Pediatric Otorhinolaryngology. 1999; 49(3):215-24. [DOI:10.1016/S0165-5876(99)00206-2]

[28] Suarez H, Angeli S, Suarez A, Rosales B, Carrera X, Alonso R. Balance sensory organization in children with profound hearing loss and cochlear implants. International Journal of Pediatric Otorhinolaryngology. 2007; 71(4):629-37. [DOI:10.1016/j. ijporl.2006.12.014] [PMID]

[29] An MH, Yi CH, Jeon HS, Park SY. Age-related changes of singlelimb standing balance in children with and without deafness. International Journal of Pediatric Otorhinolaryngology. 2009; 73(11):1539-44. [DOI:10.1016/j.jpporl.2009.07.020] [PMID]

[30] Seyedi M, Seidi F, Minoonejad H. An Investigation of the efficiency of sensory systems involved in postural control in deaf athletes and non-athletes. Journal of Sport Medicine. 2015; 7(1):111-27.

[31] Lewis S, Higham L, Cherry DB. Development of an exercise program to improve the static and dynamic balance of profoundly hearing-impaired children. American Annals of the Deaf. 1985; 130(4):278-84. [DOI:10.1353/aad.2012.1020] [PMID]

[32] Hessari FF, Norasteh AA, Daneshmandi H, Ortakand SM. The effect of 8 weeks core stabilization training program on balance in deaf students. Medicina Sportiva. 2011; 15(2):56-61. [DOI:10.2478/v10036-011-0010-4]

[33] Majlesi M, Farahpour N, Azadian E, Amini M. The effect of interventional proprioceptive training on static balance and gait in deaf children. Research in Developmental Disabilities. 2014; 35(12):3562-7. [DOI:10.1016/j.ridd.2014.09.001] [PMID]

[34] Chang YC, Hsu CT, Ho WH, Kuo YT. The effect of static balance enhance by table tennis training intervening on deaf children. International Journal of Medical and Health Sciences. 2016; 10(6):359-9.

[35] Zarei H, Rahmanpour A, Hajihoseini E. [The effects of Pilate's training on static and dynamic balance of deaf students (Persian)]. Paper presented at: $2^{\text {nd }}$ National Conference on New Finding in Sport Sciences. 15 October 2016; Tehran, Iran.

[36] Zarei $\mathrm{H}$, Norasteh AA. [Comparison of the effect of two proportionating and core stability training programs on the balance of deaf students (Persian)]. Paper presented at: First National Conference on Sport: Sport, Health, Society. 19 October 2017; Urmia, Iran.

[37] Khodashenas E, Moradi H, Asadi Ghaleni M, Heydari E, Shams $A$, Enayati $A$, et al. [The effect of selective training program on the static and dynamic balance of Deaf Children (Persian)]. Medical Journal of Mashhad University of Medical Sciences. 2017; 60(1):383-91.

[38] Zarei H, Norasteh AA, Hajihosseini E. [The effect of a combined training program on physical fitness factors among deaf boy students: a randomized clinical trial study (Persian)]. Journal of Research in Rehabilitation Sciences. 13(3):154-61.

[39] Nashner LM. Practical biomechanics and physiology of balance. In: Jacobson GP, Shepard NT, editors. Balance Function Assessment and Management. San Diego: Plural Publishing; 2014.
[40] Peterka R. Sensorimotor integration in human postural control. Journal of Neurophysiology. 2002; 88(3):1097-118. [DOI:10.1152/jn.2002.88.3.1097] [PMID]

[41] Mai JK, Paxinos G. The human nervous system. Cambridge, Massachusetts: Academic Press; 2011.

[42] Szymczyk D, Druzbicki M, Dudek J, Szczepanik M, Snela S. Balance and postural stability in football players with hearing impairment. Young Sports Science of Ukraine. 2012; 3:258-63.

[43] Potter CN, Silverman LN. Characteristics of vestibular function and static balance skills in deaf children. Physical Therapy. 1984; 64(7):1071-5. [DOI:10.1093/ptj/64.7.1071]

[44] Horak FB, Shumway-Cook A, Crowe TK, Black FO. Vestibular function and motor proficiency of children with impaired hearing, or with learning disability and motor impairments. Developmental Medicine \& Child Neurology. 1988; 30(1):6479. [DOI:10.1111/j.1469-8749.1988.tb04727.x]

[45] Peterson ML, Christou E, Rosengren KS. Children achieve adult-like sensory integration during stance at 12-years-old. Gait \& Posture. 2006; 23(4):455-63. [DOI:10.1016/j.gaitpost.2005.05.003] [PMID]

[46] Steindl R, Kunz K, Schrott-Fischer A, Scholtz A. Effect of age and sex on maturation of sensory systems and balance control. Developmental medicine and child neurology. 2006; 48(6):477-82. [DOI:10.1111/j.1469-8749.2006.tb01299.x] [PMID]

[47] Fitzpatrick R, McCloskey D. Proprioceptive, visual and vestibular thresholds for the perception of sway during standing in humans. The Journal of Physiology. 1994; 478(1):173-86. [DOI:10.1113/jphysiol.1994.sp020240] [PMID] [PMCID]

[48] Neuringer M, Jeffrey BG. Visual development: Neural basis and new assessment methods. The Journal of Pediatrics. 2003; 143(4):87-95. [DOI:10.1067/S0022-3476(03)00406-2]

[49] Brecelj J. From immature to mature pattern ERG and VEP. Documenta Ophthalmologica. 2003; 107(3):215-24. [DOI:10.1023/B:DOOP.0000005330.62543.9c] [PMID]

[50] Rinaldi NM, Polastri PF, Barela JA. Age-related changes in postural control sensory reweighting. Neuroscience Letters. 2009; 467(3):225-9. [DOI:10.1016/j.neulet.2009.10.042] [PMID]

[51] Sparto PJ, Redfern MS, Jasko JG, Casselbrant ML, Mandel EM, Furman JM. The influence of dynamic visual cues for postural control in children aged 7-12 years. Experimental Brain Research. 2006; 168(4):505-16. [DOI:10.1007/s00221-005-01098] [PMID]

[52] Cumberworth V, Patel N, Rogers W, Kenyon G. The maturation of balance in children. The Journal of Laryngology \& Otology 2007; 121(5):449-54. [DOI:10.1017/S0022215106004051] [PMID]

[53] Colledge N, Cantley P, Peaston I, Brash H, Lewis S, Wilson J. Ageing and balance: The measurement of spontaneous sway by posturography. Gerontology. 1994; 40(5):273-8. [DOI:10.1159/000213596] [PMID]

[54] Rine RM. Growing evidence for balance and vestibular problems in children. Audiological Medicine. 2009; 7(3):138-42. [DOI:10.1080/16513860903181447] 
[55] Herdman SJ, Clendaniel R. Vestibular rehabilitation. Duxbury, Vermont: FA Davis; 2014.

[56] Runge C, Shupert C, Horak F, Zajac F. Role of vestibular information in initiation of rapid postural responses. Experimental Brain Research. 1998; 122(4):403-12. [DOI:10.1007/ s002210050528] [PMID]

[57] Aali S, Rezazadeh F. [Effect of Spinal Stabilizing exercises on Lumbar Hyperlordosis by StabilizerTM Pressure Bio-feedback Unit (Persian)]. Journal of Sport Medicine Review. 2013; 5(14):135-50.

[58] Zwierzchowska A, Gawlik K, Grabara M. Energetic and coordination abilities of deaf children. Journal of Human Kinetics. 2004; 11:83-92.

[59] Zebrowska A, Gawlik K, Zwierzchowska A. Spirometric measurements and physical efficiency on children and adolescents with hearing and visual impairments. Journal of Physiology and Pharmacology. 2007; 5(Pt 2):847-57. [PMID]

[60] Houwen S, Hartman E, Visscher C. Physical activity and motor skills in children with and without visual impairments. Medicine and Science in Sports and Exercise. 2009; 41(1):103-9. [DOI:10.1249/MSS.0b013e318183389d] [PMID] 